\title{
Revealing the Influence of Molecular Chirality on Tunnel-Ionization Dynamics
}

\author{
E. Bloch, ${ }^{1}$ S. Larroque, ${ }^{1}$ S. Rozen,${ }^{2}$ S. Beaulieu, ${ }^{1}$ A. Comby $\odot,{ }^{1}$ S. Beauvarlet, ${ }^{1}$ D. Descamps,${ }^{1}$ \\ B. Fabre $\odot,{ }^{1}$ S. Petit, ${ }^{1}$ R. Taïeb, ${ }^{3}$ A. J. Uzan, ${ }^{2}$ V. Blanchet, ${ }^{1}$ N. Dudovich, ${ }^{2}$ B. Pons, ${ }^{1}$ and Y. Mairesse ${ }^{1}$ \\ ${ }^{1}$ Université de Bordeaux-CNRS-CEA, CELIA, UMR5107, F33405 Talence, France \\ ${ }^{2}$ Weizmann Institute of Science, Rehovot 76100, Israel \\ ${ }^{3}$ Sorbonne Université, CNRS, Laboratoire de Chimie Physique-Matière et Rayonnement, \\ LCPMR, F-75005 Paris, France
}

(Received 19 May 2021; accepted 13 October 2021; published 21 December 2021)

\begin{abstract}
Light-matter interaction based on strong laser fields enables probing the structure and dynamics of atomic and molecular systems with unprecedented resolutions, through high-order harmonic spectroscopy, laser-induced electron diffraction, and holography. All strong-field processes rely on a primary ionization mechanism where electrons tunnel through the target potential barrier lowered by the laser field. Tunnel ionization is, thus, of paramount importance in strong-field physics and attoscience. However, the tunneling dynamics and properties of the outgoing electronic wave packets often remain hidden beneath the influence of the subsequent scattering of the released electron onto the ionic potential. Here, we present a joint experimental-theoretical endeavor to characterize the influence of sub-barrier dynamics on the amplitude and phase of the wave packets emerging from the tunnel. We use chiral molecules, whose photoionization by circularly polarized light produces forward-backward asymmetric electron distributions with respect to the light propagation direction. These asymmetric patterns provide a background-free signature of the chiral potential in the ionization process. We first implement the attoclock technique, using bicircular two-color fields. We find that, in the tunnel-ionization process, molecular chirality induces a strong forward-backward asymmetry in the electron yield, while the subsequent scattering of the freed electron onto the chiral potential leads to an asymmetric angular streaking of the electron momentum distribution. In order to access the phase of the tunneling wave packets, we introduce subcycle gated chiral interferometry. We employ an orthogonally polarized two-color laser field whose optical chirality is manipulated on a sub-laser-cycle timescale. Numerical simulations are used to interpret the electron interference patterns inherent to this interaction scheme. They show that the combined action of the chiral potential and rotating laser field not only imprints asymmetric ionization amplitudes during the tunneling process, but also induces a forward-backward asymmetric phase profile onto the outgoing electron wave packets. Chiral light-matter interaction thus induces subtle angular-dependent shaping of both the amplitude and the phase of tunneling wave packets.
\end{abstract}

DOI: $10.1103 /$ PhysRevX.11.041056

\section{INTRODUCTION}

When randomly oriented chiral molecules are ionized by circularly polarized radiation, the angular distribution of the ejected electrons shows forward-backward asymmetries with respect to the light propagation axis [1-6]. This process, referred to as photoelectron circular dichroism (PECD), is one of the most sensitive chiroptical phenomena [7]. It can reach several tens of percents of the total signal,

Published by the American Physical Society under the terms of the Creative Commons Attribution 4.0 International license. Further distribution of this work must maintain attribution to the author(s) and the published article's title, journal citation, and DOI.
Subject Areas: Atomic and Molecular Physics, Optics Quantum Physics serving as a sensitive probe of chiral molecular structure [8,9] and dynamics [10-12]. PECD is generally seen as resulting from the scattering of the outgoing electron off the chiral potential, under the influence of the rotating ionizing electric field [13]. PECD is thus a dynamical phenomenon, emerging over a timescale of a few hundreds of attoseconds $[14,15]$.

In the single-photon ionization regime [9], the chiral photoionization dynamics is quite straightforward-the electrons directly scatter off the chiral potential during ionization. The situation can be enriched by the presence of resonances in the continuum [16,17], which affects the asymmetry of the ionization dynamics [14]. In the strongfield regime, the chiral photoionization dynamics is more complex. The electron tunnels out from the molecule and subsequently scatters off the ionic potential while being 

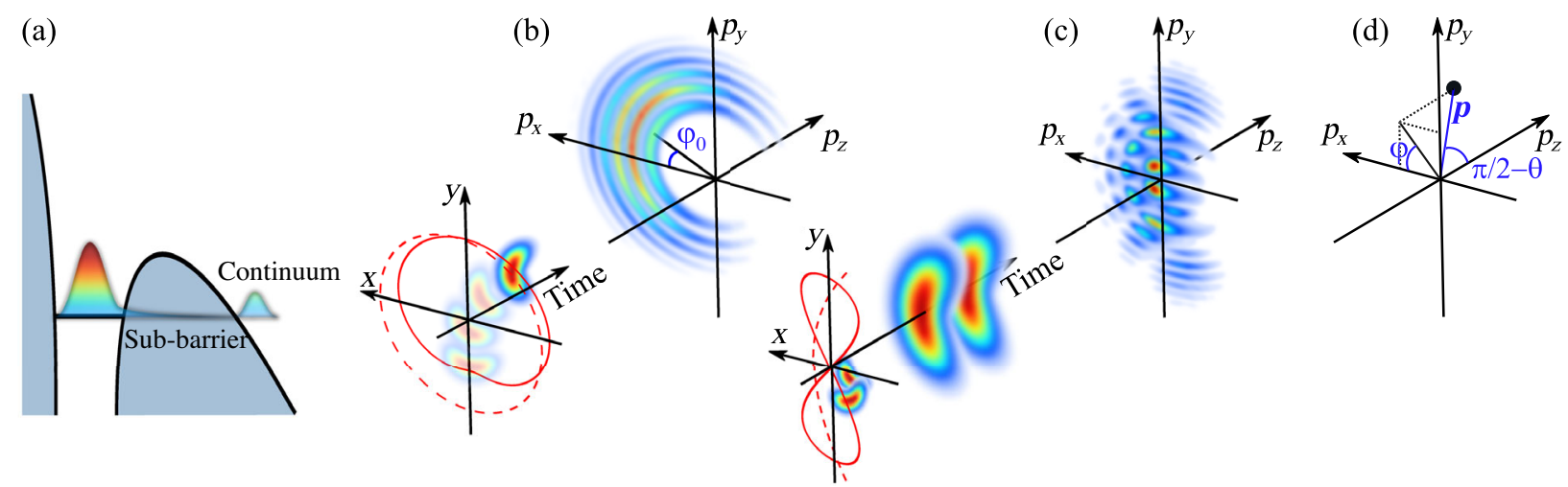

FIG. 1. Schematic view of sub-barrier and continuum electron dynamics in strong-field ionization (a) and principles of chiral attoclock (b) and subcycle gated photoelectron interferometry (c) techniques. In (a), ionization occurs as part of the initial bound electron wave packet tunnels through the target potential barrier lowered by the strong laser field. The released electron is then subject to scattering onto the ionic potential in the continuum. In (b), randomly oriented molecules are ionized by a bicircular corotating two-color laser field $\mathbf{E}(t)$ (red continuous line). The dashed red line corresponds to $-\mathbf{A}(t)$, the negative of the vector potential. In the SFA framework, the asymptotic photoelectron angular distribution [here displayed in the $\left(p_{x}, p_{y}\right)$-polarization plane] would follow the shape of $-\mathbf{A}(t)$ and point to $\varphi_{0}=0$. Deviations from this direction can be read as attoclock offsets. In the case of a chiral target, these offsets are forwardbackward asymmetric with respect to the light propagation axis $z$. In (c), the molecules are ionized by an orthogonal two-color laser field (red continuous line). Two electron wave packets are released per half laser cycle, creating an interference pattern in the photoelectron angular distribution. In the case of a chiral target, these interferences present asymmetric features which include information about the impact of chirality on the amplitude and phase profiles of tunneling electron wave packets. We display in (d) the Cartesian and spherical momentum coordinate systems used throughout the paper.

accelerated by the laser field. The dynamics can thus be decomposed in two steps: sub-barrier and into the continuum [Fig. 1(a)]. When the field is strong enough, it is common to describe the electron dynamics by means of the strong-field approximation (SFA) [18], which neglects the influence of the ionic potential on the electron acceleration in the continuum. The immediate consequence of this assumption is that no PECD should be observed in the strong-field limit [19]. Experiments [5,20] and calculations $[5,6]$ show that PECD does exist in the strong-field regime, but the dynamical origin of the asymmetry remains elusive. One may actually wonder whether the rotation of the ionizing electric field could imprint an asymmetric response under the barrier, i.e., in the tunnel-ionization dynamics of chiral molecules. Investigating this hypothesis requires disentangling the contribution of sub-barrier and continuum electron dynamics in strong-field chiral photoionization. Is such disentanglement possible? Can we obtain an insight into the chiral nature of the tunneling mechanism and rationalize its influence on the amplitude and phase properties of the emitted wave packets?

To reach that goal, we implement two complementary techniques - the chiral attoclock and subcycle gated photoelectron interferometry. In the first approach, we ionize chiral molecules using a bicircular corotating two-color laser field acting as an attoclock [21-24] [Fig. 1(b)]. The laser field rotation maps the scattering dynamics of the electrons in the continuum into angular shifts of their momentum distribution $[23,24]$. We measure forwardbackward asymmetries in this angular shift, which demonstrates the influence of molecular chirality on scattering. To discriminate unambiguously between the primary tunneling and subsequent scattering processes, we perform simulations in which the long-range chiral part of the molecular potential is screened. This screening cancels the attoclock rotation, as already established in the atomic case $[23,24]$. Surprisingly, despite the damping of the chiral continuum scattering, a large forward-backward asymmetry remains visible in the number of ejected electrons, leading to a PECD of several percent. The angularly resolved amplitude of the tunneling wave packet is, thus, strongly affected by the short-range part of the chiral potential in the sub-barrier dynamics. In the second approach, we go a step further and study the phase properties of this electron wave packet by using subcycle gated chiral interferometry. We replace the bicircular laser field by a bilinear one, combining a linearly polarized fundamental field and its orthogonally polarized second harmonic [Fig. 1(c)]. In this configuration, the electronic wave packets released by consecutive quarter optical cycles of the fundamental field interfere in momentum space [25]. These interferences encode the relative phase between the wave packets, including the phase acquired during the tunneling process [26]. The instantaneous rotation of this orthogonally polarized two-color (OTC) field temporally gates the chiral light-matter interaction on the subcycle timescale [15], allowing the separation of the sub-barrier and continuum chiral responses in the interference pattern. This study reveals that molecular chirality leads not only to a sub-barrier amplitude, but also to a sub-barrier phase 
modulation of the electronic wave packets launched by tunnel ionization. Therefore, both the attoclock and subcycle gated chiral interferometry techniques lead to the same conclusion: Tunnel ionization from chiral molecules in a rotating laser field is an enantiosensitive dynamical mechanism. Combining the two complementary measurement schemes enables us to fully disentangle the amplitude and phase of the chiral tunneling phenomenon.

\section{RESULTS}

\section{A. Principle of chiral attoclock}

The attoclock is a powerful technique to investigate the ultrafast dynamics of strong-field ionization [21,27]. It is based on the time-to-momentum mapping inherent to the SFA, where the final momentum of an electron which has tunneled out at time $t_{0}$ and does not experience any influence of the ionic potential later on, is equal to the opposite of the vector potential at time of ionization: $\mathbf{p}=-\mathbf{A}\left(t_{0}\right)$. The rotation of $\mathbf{A}(t)$ along the streaking angle $\varphi=\tan ^{-1}\left(p_{y} / p_{x}\right)$ thus acts as a clock, which points out to the ionization time $t_{0}$, a full rotation of the clock $\Delta \varphi=360^{\circ}$ corresponding to a laser period $T_{0}$. The initial implementations of the attoclock technique employed an elliptically polarized few-cycle laser field to ionize rare-gas atoms and map the ionization dynamics of the electrons into angular shifts of their momentum distribution [21,27]. This idea was later extended to other electric field shapes, releasing the constraint of using few-cycle pulses while still maintaining the time-to-momentum angular mapping [28]. Here, we use the combination of a fundamental circularly polarized laser field and its corotating second harmonic $[29,30]$, resulting in a bicircular field whose $\hat{\mathbf{x}}$ and $\hat{\mathbf{y}}$ components are defined as

$$
\begin{aligned}
& E_{x}=\sqrt{I_{\omega}} \sigma[\sin (\omega t)+\sqrt{r} \sin (2 \omega t)], \\
& E_{y}=\sqrt{I_{\omega}}[\cos (\omega t)+\sqrt{r} \cos (2 \omega t)],
\end{aligned}
$$

respectively, where $\omega$ is the fundamental laser angular frequency, $I_{\omega}$ is the fundamental intensity, $r=I_{2 \omega} / I_{\omega}$ is the ratio between the second harmonic and fundamental intensities, and $\sigma$ is the helicity of the $\omega$ and $2 \omega$ underlying fields. $\sigma=-1$ throughout the paper, unless otherwise stated. Figure 1(b) shows the shape of the bicircular field with $r=0.1$. The second harmonic induces a modulation of the electric field amplitude which maximizes at $t=0$ and minimizes at $t=T_{0} / 2, T_{0}$ being the fundamental laser period. The ionization probability thus maximizes at $t=0$.

We illustrate in Fig. 1(b) the schematic temporal evolution of an electronic wave packet launched about $t_{0}=0$. The wave packet initially points to the opposite direction of the electric field and is subsequently accelerated and streaked along $\varphi$ by the field. In the SFA framework, the wave packet would asymptotically end up centered about $\varphi_{0}=0$. Deviations from this direction, called attoclock offsets, can originate from two factors [22,31]: (i) The ionization could be delayed in time, for instance, because of a nonzero time taken by the electron to tunnel through the potential barrier [22,32], and (ii) the influence of the ionic potential on the acceleration of the electron in the continuum induces a shift of the attoclock [33]. The attoclock is thus potentially sensitive to both the sub-barrier and continuum electron dynamics. A recent theoretical study on atomic targets [23], confirmed by an experiment [24], has established that continuum dynamics is the dominant effect determining the attoclock offset.

The principle of the chiral attoclock consists in comparing the attoclock angular offsets of electrons ejected forward and backward with respect to the light propagation axis. The geometry of the interaction is depicted in Fig. 1(d). The polar angle $\theta$ describes the electron ejection angle with respect to the laser polarization plane, forward (backward) electrons being emitted at positive (negative) $\theta$. The azimuthal angle $\varphi$ still characterizes the attoclock angular streaking for fixed momentum $p$ and ejection angle $\theta$. When photoionizing a chiral molecule, we expect to measure a different number of electrons ejected forward and backward. In addition, chirality was shown to influence the angular electron streaking [20], yielding differences between the streaking angles maximizing the signal forward, $\varphi_{0}(p, \theta)$, and backward, $\varphi_{0}(p,-\theta)$. This difference is defined as the phase asymmetry $\Delta \varphi_{0}^{f / b}(p, \theta)=\varphi_{0}(p, \theta)-\varphi_{0}(p,-\theta)$.

\section{B. Chiral attoclock measurements}

For the chiral attoclock measurements, we use the $1-\mathrm{kHz}$ Aurore laser facility at CELIA [34]. The experimental setup is described in Sec. IV. Briefly, the bicircular field (at wavelengths 800 and $400 \mathrm{~nm}$ ) is produced using a MachZehnder-type interferometer, allowing an independent control of the polarization state, intensity, focal spot size, and focus position of each frequency component. The bicircular beam is focused in a gas jet in a velocity map imaging spectrometer, which records $2 \mathrm{D}$ projections of the $3 \mathrm{D}$ photoelectron angular distribution (PAD) $P(\mathbf{p})$. The bicircular field lacks the cylindrical symmetry which is necessary to retrieve $P(\mathbf{p})$ from its $2 \mathrm{D}$ projection by Abel inversion. We thus use a tomographic imaging procedure, recording a set of projections of the PAD for different orientations of the bicircular field, and reconstruct $P(\mathbf{p})$ by inverse Radon transform $[29,35]$.

Figure 2(b) shows a cut of the PAD $P(\mathbf{p})$ in the laser polarization plane, obtained by ionizing $(+)$-fenchone with a bicircular field with intensity $I_{\omega} \approx 4 \times 10^{13} \mathrm{~W} \cdot \mathrm{cm}^{-2}$ and ratio $r \approx 0.1$. In the strong-field regime, ionization mostly proceeds from the highest occupied molecular orbital (HOMO), since the ionization probability exponentially decreases as the ionization potential increases, which disfavors electron ejection from inner shells. The PAD shows several above-threshold ionization (ATI) [36] peaks, attributed to tunnel ionization from the HOMO, which 
(a)
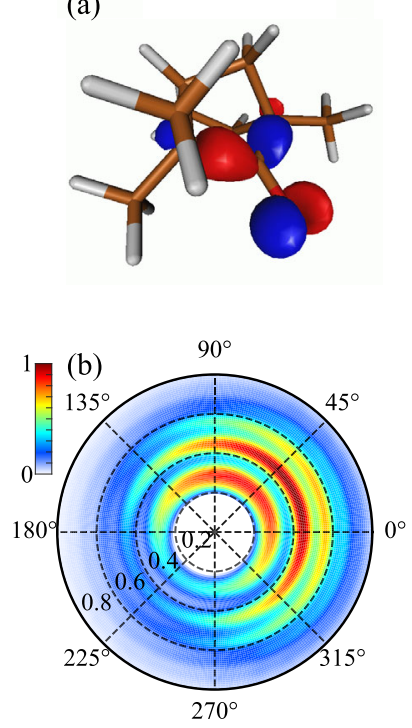

(f)
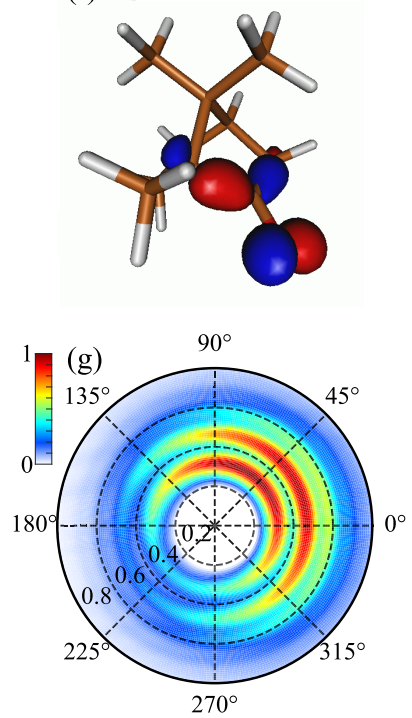
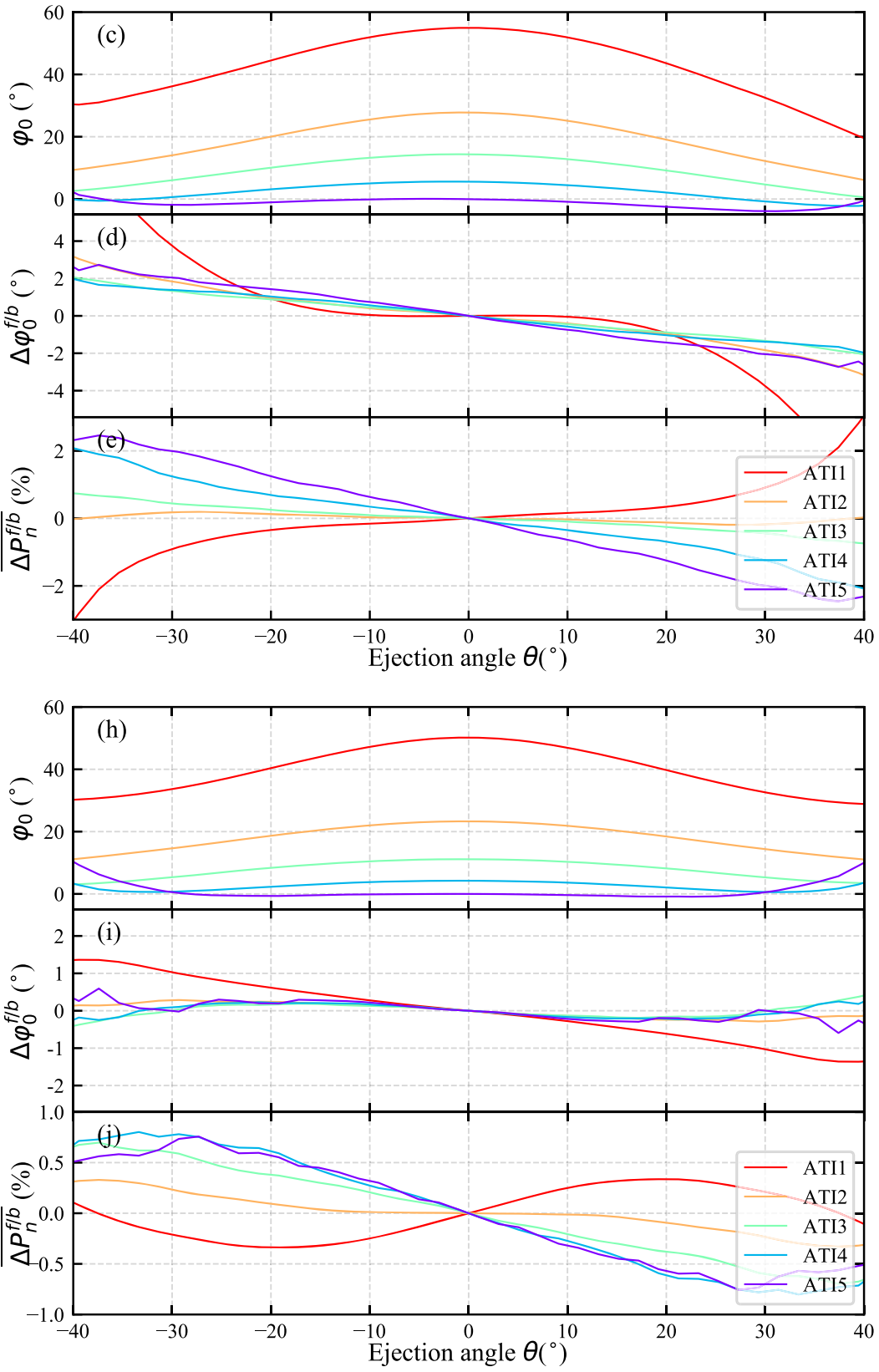

FIG. 2. Chiral attoclock measurements in (+)-fenchone and (+)-camphor, using an 800- and 400-nm bicircular field with $I_{\omega} \approx$ $4 \times 10^{13} \mathrm{~W} \cdot \mathrm{cm}^{-2}$ and $r \approx 0.1$. (a) Molecular structure and highest occupied molecular orbital of fenchone obtained by means of Hartree-Fock calculations. (b) Photoelectron angular distribution from fenchone in the laser polarization plane $(\theta=0)$. The radius is the photoelectron momentum in a.u. (c) Attoclock streaking angle $\varphi_{0, n}$ for ATI peaks $n=1-5$ (from red to purple) as a function of the electron ejection angle $\theta$. For each ATI peak $n$, we display in (d) the forward-backward asymmetry in the attoclock streaking angle $\Delta \varphi_{0, n}^{f / b}(\theta)$ and in (e) the forward-backward asymmetry in electron yield $\overline{\Delta P_{n}^{f / b}}(\theta)$. (f)-(j) are identical to (a)-(e) but relative to $(+)$-camphor.

maximize at different streaking angles $\varphi_{0}$. To extract these angles, we integrate each ATI peak of the order of $n$ over its full momentum width to obtain a polar signal as a function of the streaking angle $\varphi$. The maximizing values $\varphi_{0, n}$ are then extracted for each ejection angle $\theta$ of the electrons with respect to the polarization plane [Fig. 2(c)]. Since the absolute orientation of the bicircular field is undetermined in the experiment, we arbitrarily set it by assuming that $\varphi_{0, n}(\theta=0)=0$ for the highest ATI peak, consistently with SFA calculations. Around $\theta=0$, the first ATI peak is offset by approximately $60^{\circ}$ with respect to the highest one. The streaking angle varies by almost $40^{\circ}$ between electrons ejected in the laser polarization plane and about $\theta=40^{\circ}$. To the best of our knowledge, the dependency of the attoclock 
streaking as a function of the electron ejection angle with respect to the polarization plane has never been investigated. This observable brings a deeper insight on the ionization dynamics, in a similar manner as the angleresolved photoionization delays [14,37]. As the electron kinetic energy increases, the streaking angle as well as its variation with electron ejection angle decrease.

In order to study the influence of chirality on the ionization dynamics, we investigate the subtle asymmetry of $\varphi_{0, n}(\theta)$. The forward-backward asymmetric streaking phase $\Delta \varphi_{0, n}^{f / b}$ is hardly visible by eye in Fig. 2(c), but it can be extracted numerically as represented in Fig. 2(d). ATI peaks $2-5$ show a remarkably similar behavior, with a close-to-linear dependency of $\Delta \varphi_{0, n}^{f / b}$ which reaches $-3^{\circ}$ at $\theta=40^{\circ}$. The first ATI peak shows a more complex evolution and reaches higher values. Our measurement demonstrates that the attoclock streaking angle is forwardbackward asymmetric in chiral molecules, as previously observed in Ref. [20]. We confirm in Sec. IV that $\Delta \varphi_{0, n}^{f / b}$ is a genuine chiral observable, since it reverses sign when switching from (+)- to (-)-fenchone.

Chiral photoionization produces not only asymmetric streaking angles, but also a forward-backward asymmetry in the amount of photoelectrons ejected forward and backward. This asymmetry is defined by $\Delta P^{f / b}(\mathbf{p})=$ $2[P(p, \theta, \varphi)-P(p,-\theta, \varphi)] /[P(p, \theta, \varphi)+P(p,-\theta, \varphi)]$ at each point $\mathbf{p}$ of the momentum space. It is convenient to introduce the averaged asymmetry $\overline{\Delta P_{n}^{f / b}}(\theta)$ of electrons belonging to the $n$th ATI peak and ejected along the polar direction $\theta$ with respect to the polarization plane. $P(\mathbf{p})$ is integrated over $\varphi$ and summed over the radial momentum width of the ATI peak to yield $\overline{P_{n}}(\theta) \cdot \overline{\Delta P_{n}^{f / b}}(\theta)$ is then defined as $2\left[\overline{P_{n}}(\theta)-\overline{P_{n}}(-\theta)\right] /\left[\overline{P_{n}}(\theta)+\overline{P_{n}}(-\theta)\right]$. Averaged asymmetries are reported in Fig. 2(e) for the five ATI peaks. The asymmetry from the first ATI ring is opposite to the other ones. This peculiar behavior is discussed later on. For ATI2-5, $\overline{\Delta P_{n}^{f / b}}(\theta)$ behaves almost linearly as a function of the electron ejection angle and monotonically increases with increasing kinetic energy of the electrons. $\overline{\Delta P_{5}^{f / b}}(\theta)$ reaches $-2.4 \%$ around $\theta=40^{\circ}$.

To demonstrate that the differential $\Delta \varphi_{0, n}^{f / b}$ angles are general chiral features, we repeat the measurements in camphor, a structural isomer of fenchone. These two isomers are often used to illustrate the structural sensitivity of PECD measurements [3,38,39]. Figures 2(h)-2(j) show the chiral attoclock measurements in $(+)$-camphor. The streaking angles are remarkably similar to those obtained in fenchone, exhibiting only slightly smaller values. This is consistent with the conclusions of Torlina et al. [23] — the attoclock is mostly sensitive to the long-range part of the molecular potential, which is similar in ionized camphor and fenchone.
The forward-backward asymmetry in the attoclock streaking angles [Fig. 2(i)] and ATI yields [Fig. 2(j)] is more sensitive to structural isomerism. The differential streaking angles $\Delta \varphi_{0, n}^{f / b}$ are much weaker in camphor than in fenchone-they reach nonzero values only for the first ATI peak. The averaged asymmetries $\overline{\Delta P_{n}^{f / b}}(\theta)$ behave differently as a function of $\theta$ in the two isomers. However, they present a similar trend with electron kinetic energy: $\overline{\Delta P_{n}^{f / b}}(\theta)$ switches sign between $n=1$ and 2 and then increases with increasing ATI order. This behavior contradicts our intuition of chiral scattering, whose influence is expected to vanish as the electron kinetic energy increases. In this respect, the PECD associated to ionization from a given molecular orbital is known to decrease as the electron energy increases in the multiphoton regime $[5,6,40]$ as well as in standard single-photon ionization far from threshold $[13,38]$. The distinct behavior observed in the present measurements is linked to the ionization regime associated to our experiments. The Keldysh parameter in our experimental conditions is $\gamma \approx 1.3$, which corresponds to the nonadiabatic tunneling regime. In this ionization regime, the influence of the ionic potential on the departing electron is expected to be weak. The potential does have some influence on the attoclock, as demonstrated by the nonzero differential streaking angles, but this influence is attenuated because the attoclock shift is mostly monitored by the longrange part of the potential. By contrast, our measurements reveal a large forward-backward asymmetry in the number of ejected electrons, which further increases with increasing electron kinetic energy. What is the origin of this exacerbated asymmetry? Is the electron yield more sensitive to the long-range chiral scattering than the angular streaking? Or does this observation indicate that the yield is already asymmetric at the exit of the tunnel, carrying the signature of sub-barrier dynamics? To answer these questions, we develop a theoretical model of the interaction, described in the next section.

\section{Chiral attoclock calculations}

To shed light on the origin of the forward-backward asymmetry of electron yield and angular streaking in chiral strong-field photoionization, we perform quantummechanical calculations solving the time-dependent Schrödinger equation (TDSE) for the strong-field ionization of a toy-model chiral molecule by a bicircular field (see Sec. IV). This fictitious molecule consists of a single electron evolving in the field of four nuclei whose effective charges and positions are set to yield an ionization potential of $9 \mathrm{eV}$, similar to that of fenchone. Figure 3(a) illustrates the ionic potential of the toy model in the $(x, y)$ plane. Figure 3(b) shows the PAD obtained when the toy molecule is ionized by a bicircular field with $I=2 \times 10^{13} \mathrm{~W} \cdot \mathrm{cm}^{-2}$ and $r=0.1$. The intensity employed in the calculations is 

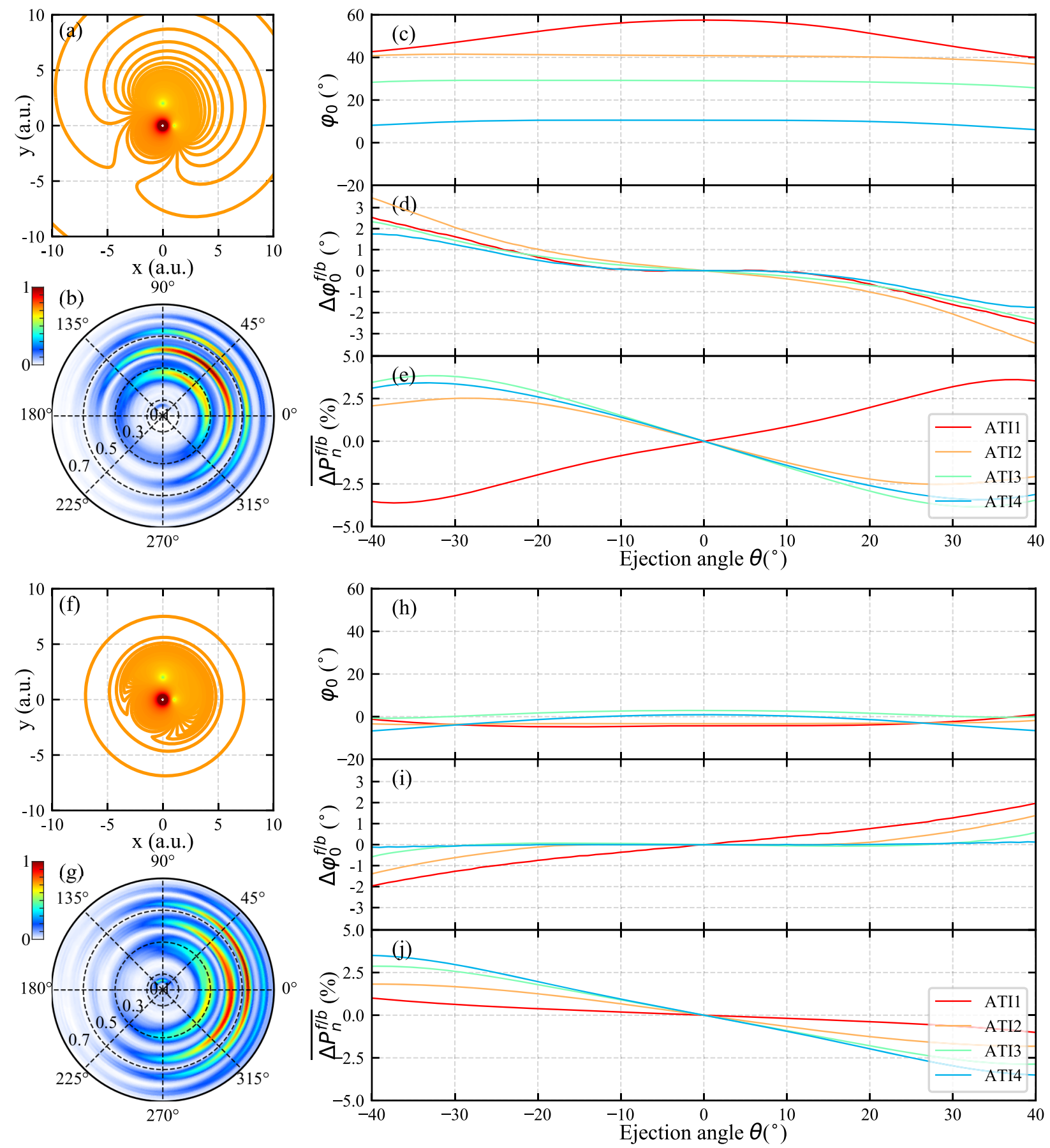

FIG. 3. Chiral attoclock TDSE calculations in a toy-model chiral molecule, using an 800- and 400-nm bicircular field with $I_{\omega}=$ $2 \times 10^{13} \mathrm{~W} \cdot \mathrm{cm}^{-2}$ and $r=0.1$. (a) Potential of the toy-model molecule in the $\theta=0$ plane. (b) Photoelectron angular distribution in the laser polarization plane $(\theta=0)$. The radius is the photoelectron momentum in a.u. (c) Attoclock streaking angle $\varphi_{0}(\theta)$ of the first four ATI peaks (from red to blue) as a function of the electron ejection angle $\theta$. (d) Forward-backward asymmetry in the attoclock streaking angles $\Delta \varphi_{0, n}^{f / b}$. (e) Forward-backward asymmetry in the intensity of the first four ATI peaks (from red to blue). (f)-(j) are the same as (a)-(e) but relative to a Yukawa-screened molecule.

somewhat lower than the experimental one. In practice, this is the highest intensity for which the calculations could be converged with our current computational capabilities, as discussed in Sec. IV. Four ATI peaks of significant magnitude show up in the computed PAD, the first one, centered about $p=0.25$ a.u., being noticeably structured along the streaking direction $\varphi$. We thus define the mean streaking angle $\varphi_{0, n}(\theta)$ of each ATI peak $n$ as the center of 
mass of the ATI electron distribution along $\varphi$. The chiral angular streaking asymmetries $\Delta \varphi_{0, n}^{f / b}(\theta)$ as well as the asymmetries $\overline{\Delta P_{n}^{f / b}}(\theta)$ in electron yields are then defined as in the experiment.

The theoretical results are in good qualitative agreement with the measurements. The streaking angles $\varphi_{0, n}(\theta)$ decrease with increasing order of the ATI peak. This indicates a smaller influence of the molecular potential on higher ATI peaks, which is intuitive, since the scattering waves associated to high-energy electrons tend to plane waves, insensitive to the potential. The streaking angles are slightly forward-backward asymmetric, leading to a $\Delta \varphi_{0, n}^{f / b}$ which reaches approximately $-3.5^{\circ}$ at $\theta=40^{\circ}$ for ATI2 and decreases for higher ATI peaks. $\overline{\Delta P_{n}^{f / b}}(\theta)$ changes sign between $n=1$ and $n=2$ and increases between ATI2 and ATI3-4, where it reaches $-4 \%$ at $\theta \sim 40^{\circ}$. The general trends of the three observables are similar to those observed experimentally in fenchone and camphor. This observation is remarkable given the difference between the model and the actual structure of these molecules, pointing toward a universal behavior of asymmetric features in strong-field ionization of chiral molecules.

The particular behavior of the first ATI peak, mainly appearing in Figs. 3(e), 2(e), and 2(j) in terms of $\overline{\Delta P_{n}^{f / b}}(\theta)$ sign reversal between $n=1$ and $n=2$, can be related to continuum scattering effects which depend on the energy of the ejected electron. Low-energy electrons are indeed emitted at times where the field has almost maximal amplitude. Therefore, they exit from the tunnel at rather short distances and are subsequently subject to significant scattering off the chiral potential. As is well known from one-photon PECD dynamics [13], this chiral scattering can lead to opposite preferential directions of electron emission over a small kinetic energy range, as the one encompassing ATI1 and ATI2. Higher-energy electrons emerge from the tunnel at larger distances where the chiral continuum scattering gradually vanishes.

In order to disentangle sub-barrier and continuum effects, we perform a second TDSE calculation following the idea proposed by Torlina et al. in the atomic attoclock case, which consists in screening the long-range part of the potential [23]. The nuclear skeleton of our toy molecule extends up to 3 a.u. We thus damp the long-range chiral potential beyond a radial distance $r_{0}=3.5$ a.u. by multiplying it by an isotropic cutoff Yukawa term $\exp ^{-\left(r-r_{0}\right)}$ for $r>r_{0}$. The screened potential is illustrated in Fig. 3(f). It vanishes for radial distances $r \gtrsim 8$ a.u. out of the molecular core. The results of the attoclock calculations for the screened potential are depicted in Figs. 3(g)-3(j). Cutting off the long-range part of the chiral potential has a large influence on the attoclock streaking angles. All ATI peaks point around the horizontal direction in Fig. 3(g), and the streaking angles remain small for all ejection angles.
The situation is thus close to SFA calculations which lead to exactly zero streaking angles [23]. The differential attoclock angles $\Delta \varphi_{0, n}^{f / b}(\theta)$ switch sign with respect to those obtained with an unscreened potential. However, they are significantly attenuated, except for the first two ATI peaks because of incomplete screening in the region where these electrons scatter just after tunneling.

These results demonstrate the overall efficiency of Yukawa screening in damping the influence of the molecular potential on electron scattering. Surprisingly however, Fig. 3(j) shows that the screened molecule exhibits a significant forward-backward asymmetry in the electron yield. The first ATI peak does not present an odd behavior anymore, and $\overline{\Delta P_{n}^{f / b}}(\theta)$ increases with increasing ATI order $n$, reaching $-4 \%$ at $\theta=40^{\circ}$ - the same value as in the nonscreened molecule. Such an asymmetry in the photoelectron angular distribution thus necessarily emerges from the sub-barrier dynamics: Despite the random molecular orientations, the electrons ending up in the backward hemisphere are favored by tunnel ionization compared to the electrons ending up forward.

In addition to the amplitude asymmetry in the photoelectron angular distribution, the asymmetric tunneling could also result in a forward-backward asymmetry in the phase of the ejected electron wave packets, which is not resolved by the above measurement. Measuring and resolving this sub-barrier phase would provide a complete insight on the influence of chirality on the tunneling dynamics. In the next part, we show that such a measurement can be performed by subcycle gated chiral interferometry, using an orthogonal two-color laser field.

\section{Chiral photoionization by orthogonal two-color laser fields}

Resolving the phase of an electron wave packet requires an interferometric measurement. Photoelectron interference is very common in strong-field ionization [41], leading to important processes such as photoelectron holography $[42,43]$ or laser-induced electron diffraction [44-46]. When the ionizing pulse is linearly polarized, two arms of an interferometer are naturally built by the ionization dynamics. The first arm corresponds to electrons ionized between the zero and the following peak of the fundamental field. These electrons directly escape the target under the influence of the field. The second arm consists of electrons ionized between the peak and the next zero of the fundamental field. Their trajectory is indirect, being first accelerated in one direction before reversing. Within the strong-field approximation, electrons from the two families end up with the same final momentum, producing an interference pattern in momentum space [see Fig. 1(c)].

Performing chiral-sensitive photoelectron interferometry measurements is not straightforward. The angular streaking of the photoelectrons by a rotating electric field prevents 


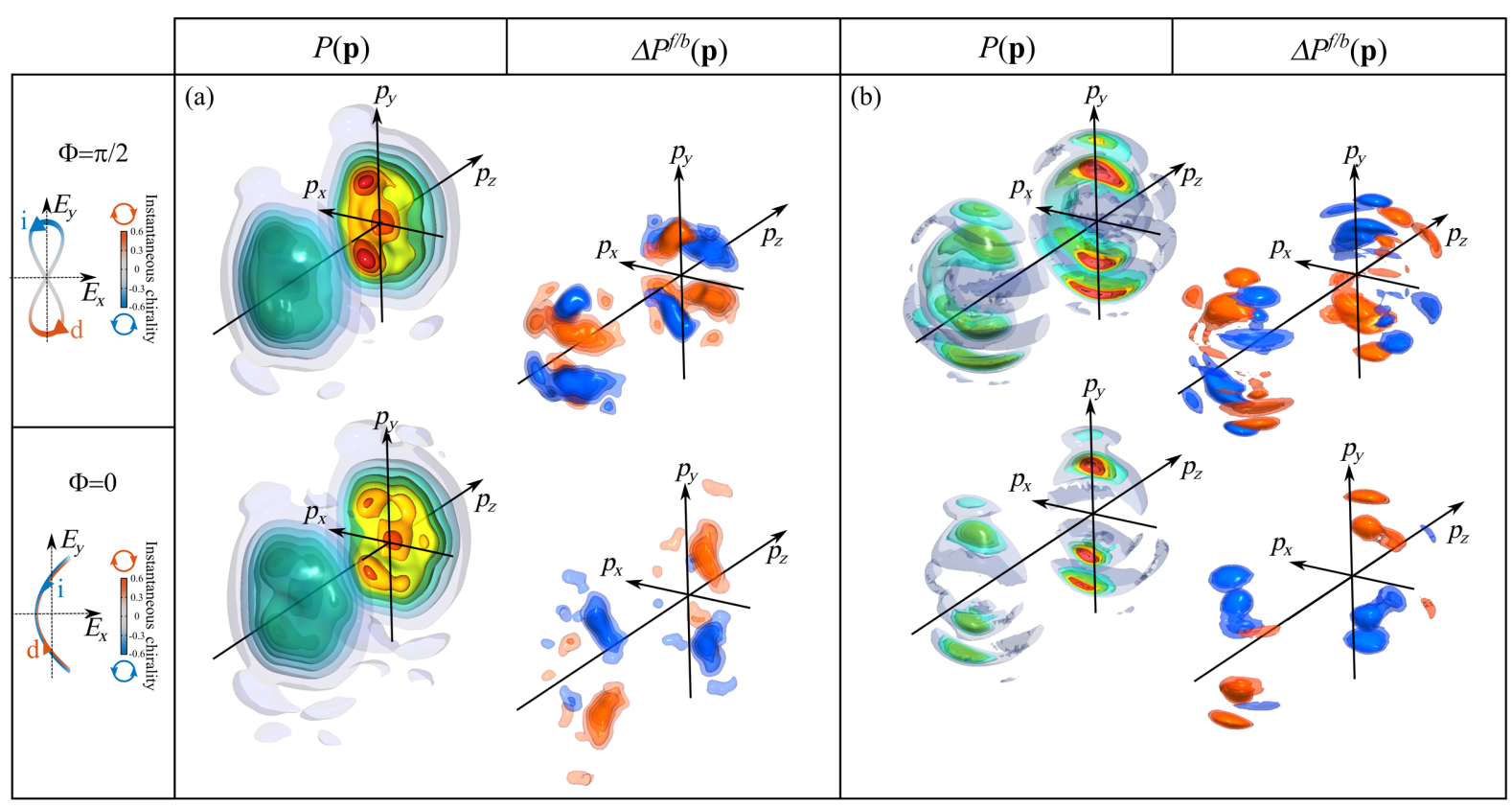

FIG. 4. Measurements (a) and calculations (b) of chiral photoionization by an OTC field. (a) Measured photoelectron angular distribution $P(\mathbf{p})$ and forward-backward asymmetry $\Delta P^{f / b}(\mathbf{p})$ from $\left(+\right.$ )-fenchone ionized by an OTC field with $I_{\omega} \approx 5 \times 10^{12} \mathrm{~W} \cdot \mathrm{cm}^{-2}$, $r \approx 0.1, \phi=\pi / 2$ (8-shaped field) and $\phi=0$ (C-shaped field). (b) Corresponding TDSE calculations in the toy-model molecule.

the observation of interference in circularly polarized light - the electrons released during consecutive quarter cycles of the field do not overlap in momentum space and, thus, do not interfere. The situation is similar with the corotating bicircular field used in our attoclock scheme. To solve this issue, we use an OTC laser field. In contrast to the bicircular case, the OTC field creates interference patterns while still presenting a nonzero instantaneous optical chirality. Combining the chiral property of circularly polarized light with the interference features inherent to the linear polarization, we create a scheme which enables us to study the phase properties of the chirosensitive photoelectron interference.

An OTC field $\mathbf{E}$ is obtained by superimposing a fundamental field $E_{\omega} \cos (\omega t) \hat{\mathbf{y}}$ and its orthogonally polarized second harmonic $E_{2 \omega} \cos (2 \omega t+\phi) \hat{\mathbf{x}}$ :

$$
\mathbf{E}=E_{0} \cos (\omega t) \hat{\mathbf{y}}+E_{0} \sqrt{r} \cos (2 \omega t+\phi) \hat{\mathbf{x}},
$$

where $E_{0}=E_{\omega}, r=I_{2 \omega} / I_{\omega}$ is the intensity ratio between the two components, and $\phi$ is their relative phase. The vectorial temporal evolution of the laser field can be tailored from describing an " 8 " shape $(\phi=\pi / 2 \pm \pi)$ to a "C" $(\phi=0 \pm \pi)$ shape (Fig. 4). The vector potentials associated with these two cases have, respectively, $\mathrm{C}$ and 8 shapes.

The electric field of an OTC field rotates in time, but its helicity reverses every half laser cycle. The time-dependent field rotation is described by the instantaneous optical chirality $C(t)$ [47]:

$$
C(t)=C_{0}\left[E_{x}(t) \partial_{t} E_{y}(t)-E_{y}(t) \partial_{t} E_{x}(t)\right],
$$

where $C_{0}$ is a normalization factor defined such that $C(t)=1$ for a right circularly polarized field. The evolution of the instantaneous chirality of an OTC field is shown in Fig. 4(a) for different relative phases between the vectorial components of the field.

Figure 4(a) shows the experimental 3D photoelectron angular distributions $P(\mathbf{p})$ and forward-backward asymmetries resulting from the photoionization of $(+)$-fenchone by OTC fields with $I_{\omega} \approx 5 \times 10^{12} \mathrm{~W} \cdot \mathrm{cm}^{-2}$ and $r \approx 0.1$. The fundamental laser pulse used in this experiment is a 135 -fs pulse at $1030 \mathrm{~nm}$ provided by the BlastBeat $\mathrm{Yb}$ :fiber laser system operating at $166 \mathrm{kHz}$. The 3D photoelectron momentum distributions are collected using a cold target recoil ion momentum spectrometer (COLTRIMS). Details on the experimental setup and acquisition procedure are given in Sec. IV.

The Keldysh parameter associated to the interaction is $\gamma \approx 3$. This indicates that ionization takes place in the multiphoton regime, yet approaching the nonadiabatic tunneling regime characterized by $\gamma \sim 1$. Unfortunately, experiments could not be conducted using stronger fields because of artifacts in the photoelectron imaging at high laser intensity.

When the relative two-color phase $\phi$ is set to $\pi / 2$, the driving field $\mathbf{E}(t)$ is 8-shaped, and the photoelectron angular distribution shows a clear asymmetry along the $p_{x}$ axis. The overall shape of this distribution tends to reproduce the inverted $\mathbf{C}$ shape of $-\mathbf{A}(t)$. Other measurements (not shown 
for the sake of conciseness) confirm that the 3D photoelectron angular distribution presents an approximate C shape when the relative phase between the OTC components is switched to $\phi=3 \pi / 2$. The distribution thus features some of the characteristics of strong-field ionization, even though $\gamma \gtrsim 1$.

The 3D asymmetry in electron yield, displayed in Fig. 4(a), is here defined as $\Delta P^{f / b}(\mathbf{p})=2[P(p, \theta, \varphi)-$ $P(p,-\theta, \varphi)] / P_{\max }$, where $P_{\max }$ is the maximal value of $P(\mathbf{p})$. For $\phi=\pi / 2$, the experimentally resolved 3D forwardbackward asymmetry shows fringes of alternating sign on the main ATI sphere. This indicates that interferences are at play in the ionization process and that they preferentially show up in the asymmetric part of the electron distribution. As theoretically predicted [48] and observed in 2D measurements using velocity map imaging [15], the forwardbackward asymmetry is antisymmetric along the polarization direction of the fundamental field $p_{y}$. This reflects the opposite optical chirality experienced by electrons ending up in the upper and lower hemispheres, as shown in Fig. 4(a). When $\phi$ is set to 0 , the 3D photoelectron angular distribution is more symmetric along $p_{x}$ than in the $\phi=\pi / 2$ case. This is quite consistent with the 8 shape of the vector potential. The forward-backward asymmetry is highly localized in momentum space along $p_{y}$, and interference patterns are of smaller amplitude than in the $\phi=\pi / 2$ case.

Figure 4(b) shows the results of TDSE calculations, performed using the toy-model molecule introduced in the previous section, in the same conditions as the experiment. The results are in good qualitative agreement with the measurements on fenchone. The shape of the photoelectron angular distributions looks like the form of $-\mathbf{A}(t)$, and the $3 \mathrm{D}$ forward-backward asymmetry is strongly sensitive to the two-color relative phase $\phi$. This latter is highly structured for $\phi=\pi / 2$, while it is localized along $p_{y}$ for $\phi=0$.

This study leads us to draw three conclusions. First, the 3D chiral signal is extremely sensitive to the relative phase between the components of the OTC field. The temporal evolution of the instantaneous chirality plays a crucial role in the photoionization dynamics, acting as a subcycle gate. This scheme constitutes a time-gated version of PECD, which we refer to as enantiosensitive subcycle antisymmetric response gated by electric-field rotation (ESCARGOT) [15]. Second, the appearance of fringes when the OTC field is 8-shaped suggests that the chiroptical signal carries an enhanced signature of interference effects. Third, the good agreement between experiment and theory confirms the ability of our simple model to faithfully describe the interaction of a chiral system with OTC fields. In the following, we rely on this agreement to pursue our investigation of chiral interferometry in the genuine strong-field regime, characterized by $\gamma \leq 1$, from a theoretical point of view.

\section{E. Chiral interferometry}

We have seen in the first section that the influence of chirality on the continuum electron dynamics is strongly attenuated in a Yukawa-screened toy molecule, enabling us to isolate the role of sub-barrier dynamics. We thus use these molecules as our target $\left(I_{p}=7.2 \mathrm{eV}\right)$ and study their ionization by an OTC field with $I_{\omega}=5 \times 10^{13} \mathrm{~W} \cdot \mathrm{cm}^{-2}$ and $r=0.1$. Before presenting the results of the TDSE calculations, we build an intuitive model based on SFA, which aims at understanding the role of continuum and subbarrier chiral dynamics in photoelectron interferometry.

Figure 5(a) illustrates the birth of direct and indirect electron wave packets, obtained by SFA in the conditions of the TDSE calculation. These wave packets are initially delayed, but they have asymptotically the same momentum distribution, which reflects the $\mathrm{C}$ shape of the vector potential since $\mathbf{p}=-\mathbf{A}\left(t_{0}\right)$ in the SFA. Their coherent superposition thus yields an interference pattern in the total PAD displayed in Fig. 5(a) (see also Ref. [49]). The PAD is further radially structured due to intercycle interferences producing ATI peaks. When the relative phase between the two components of the OTC field is changed to $\phi=0$, the OTC field has a C-shape, while the vector potential describes an 8 shape. The electrons from the two families are then streaked in opposite directions along the polarization direction of the second-harmonic field, which lowers the contrast of the interference pattern [25]. However, clear fringes remain visible in the momentum distribution, as shown in Fig. 5(b).

To evaluate how the subcycle chirality can influence the interference pattern, we use a qualitative approach by introducing a perturbative chiral sensitivity into the SFA calculations. We mimic the combined effect of the chiral light and chiral molecular potential on the ionization probability by introducing $p_{z}$-antisymmetric amplitude $(\alpha)$ and phase $(\beta)$ perturbations to the SFA electron quantum paths:

$$
P(\mathbf{p})=\left|\sum_{j}\left[1+\alpha\left(\mathbf{p}, t_{0}^{j}\right)\right] e^{i\left[S\left(\mathbf{p}, t_{0}^{j}\right)+\beta\left(\mathbf{p}, t_{0}^{j}\right)\right]}\right|^{2},
$$

where $j=d$ and $i$ for direct and indirect electrons, respectively, and

$$
\begin{aligned}
& \alpha=\alpha_{0} \operatorname{sgn}\left(p_{z}\right) \int_{t_{0}^{j}}^{+\infty} C(t) R\left(t-t_{0}^{j}\right) d t, \\
& \beta=\beta_{0} \operatorname{sgn}\left(p_{z}\right) \int_{t_{0}^{j}}^{+\infty} C(t) R\left(t-t_{0}^{j}\right) d t .
\end{aligned}
$$

Here, $\alpha_{0}$ and $\beta_{0}$ quantify the chiral-induced amplitude and phase, respectively, $R(t)$ is the response function of the system to the light chirality $C(t)$, and $t_{0}^{j}$ is the ionization time of electron $j . \alpha_{0}$ and $\beta_{0}$ are set to 0.1 to fulfill perturbative constraints. The chiroptical response shows up through the 

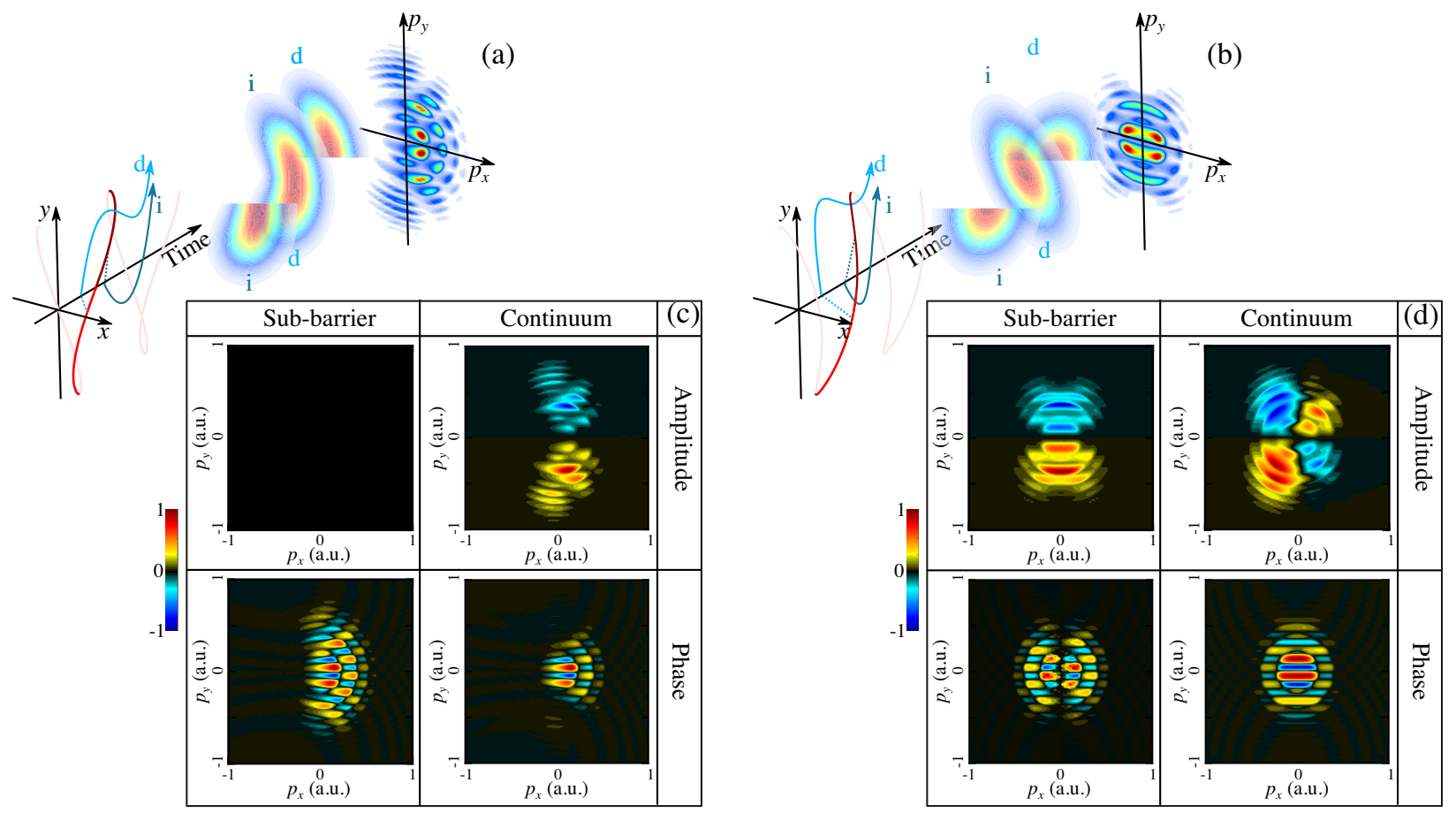

FIG. 5. Chiral strong-field photoelectron interferometry. (a) Schematic drawing of direct " $d$ " and indirect " $i$ " electron paths associated to the ionization of a target with $7.2 \mathrm{eV}$ ionization potential by an 8 -shaped OTC field (shaded red line) with $I_{\omega}=5 \times 10^{13} \mathrm{~W} \cdot \mathrm{cm}^{-2}$ and $r=0.1$. These electron paths belong to delayed wave packets whose asymptotic momentum shapes are reported in terms of shaded contour plots (SFA calculations). The direct and indirect wave packets finally interfere, yielding a clear angular fringe pattern on the electron detector. (c) Projected forward-backward asymmetries $\Delta \mathcal{P}^{f / b}\left(p_{x}, p_{y}\right)$ modeled by the chiral-SFA approach, assuming different sources of chiral response - amplitude vs phase effects coupled to sub-barrier vs continuum dynamics. These asymmetries are arbitrarily normalized. (b) and (d) are the same as (a) and (c) but relative to a C-shaped OTC field.

3D forward-backward asymmetry whose unnormalized form $\Delta P^{f / b}(\mathbf{p})=P(p, \theta, \varphi)-P(p,-\theta, \varphi)$ is used in the following unless otherwise specified. For the sake of clarity of illustrations, it can be convenient to introduce the PAD and related asymmetry projected onto the polarization plane. The projected PAD, $\mathcal{P}\left(p_{x}, p_{y}\right)$, is simply computed as $\mathcal{P}\left(p_{x}, p_{y}\right)=\int P(\mathbf{p}) d p_{z}$, and the corresponding forwardbackward asymmetry is then $\Delta \mathcal{P}^{f / b}\left(p_{x}, p_{y}\right)=\mathcal{P}_{+}\left(p_{x}, p_{y}\right)-$ $\mathcal{P}_{-}\left(p_{x}, p_{y}\right)$, where $\mathcal{P}_{+(-)}\left(p_{x}, p_{y}\right)=\int_{0(-\infty)}^{+\infty} P(\mathbf{p}) d p_{z}$.

We consider two scenarios where the chiral response is introduced either in the sub-barrier or in the continuum scattering process. In the first case, the perturbation is imprinted by tunneling. The response is, thus, instantaneous, described by a Dirac function $R(t)=\delta\left(t-t_{0}^{j}\right)$. This approach is justified by the recent theoretical investigation of interference patterns in photoelectron spectra from atoms, which has shown that adding a sub-barrier Coulomb correction to SFA calculations reproduces the results of TDSE calculations [50]. Figure 5(c) shows the projected forward-backward asymmetries $\Delta \mathcal{P}^{f / b}\left(p_{x}, p_{y}\right)$ produced by tunneling amplitude and phase perturbations, for an 8-shaped electric field $(\phi=\pi / 2)$. The instantaneous optical chirality changes sign between the time intervals $\left[0, T_{0} / 4\right]$ and $\left[T_{0} / 4, T_{0} / 2\right]$ [see Fig. 4(a)]. As a consequence, the amplitude modulations experienced by indirect and direct electrons, which are born in these respective time ranges, are opposite, and the resulting asymmetry is zero. By contrast, an opposite phase shift of direct and indirect electrons shifts the interference patterns in opposite directions, resulting in a significant asymmetry.

In the second scenario, the forward-backward asymmetry is imprinted by scattering during the acceleration of the electron in the continuum. In contrast to the previous case, the chiral response is not instantaneous. We assume that it decays with increasing electron distance from ionic core, mimicking the limited spatial range of the chiral potential: $R(t)=\exp \left\{-4 \ln (2)\left[r(t) / r_{0}\right]^{2}\right\}$, with a full width at half maximum $r_{0}=2 \mathrm{~nm}$. Figure $5(\mathrm{c})$ shows that, within this model, a continuum amplitude modulation produces a significant asymmetry. This is due to the fact that, even if direct and indirect electrons experience opposite optical chiralities at $t_{0}$, the accumulated chiralities along their trajectories are approximately similar [15]. A scattering phase asymmetry results in a forward-backward asymmetry pattern $\Delta \mathcal{P}^{f / b}\left(p_{x}, p_{y}\right)$ similar to the one produced by subbarrier phase effects, although it vanishes at high values of $p_{y}$ in Fig. 5(c). 
A better discrimination between the sub-barrier and continuum chiral effects can be obtained by changing the relative phase between the two components of the OTC field to produce a $\mathrm{C}$-shaped field. This configuration leads to a smaller region of interference, due to the opposite lateral streaking of electrons from the two families, but it is best suited to unravel the origin of the chiral response. Indeed, Fig. 5(d) shows that the four types of chiral perturbations, involving sub-barrier vs scattering dynamics and amplitude vs phase effects, produce chiral responses with drastically different fingerprints. This illustrates how subcycle gated chiral interferometry is sensitive not only to the timescale of the chiral response but also to the underlying properties of the ionizing wave packets. Measuring the forward-backward asymmetry of the subcycle gated interference pattern can, thus, reveal the dynamical origin of the chiral response, as well as retrieve its amplitude and phase.

\section{F. Determination of the chiral response}

We remedy the lack of 3D-resolved experimental data on strong-field ionization by OTC fields by analyzing the TDSE calculations. Our previous attoclock calculations have shown that the asymmetry in electron yield is encoded within the tunneling ionization step. We now aim at observing the amplitude and phase profiles of the electron chiral wave packets at the exit of the tunnel. Therefore, we consider our Yukawa-screened toy molecule where the long-range part of the potential is damped. This damping favors the influence of tunneling over scattering, even though the latter may still show residual effects, as seen in Fig. 3(i) in terms of small but nonzero chiral attoclock streaking angles.

Figures 6(a) and 6(e) show projections of the PADs obtained by means of TDSE calculations (detailed in Sec. IV) in the polarization plane of 8- and C-shaped fields with $I=5 \times 10^{13} \mathrm{~W} \cdot \mathrm{cm}^{-2}$ and $r=0.1$. The overall agreement of these PADs with their SFA counterparts, displayed in Figs. 6(c) and 6(g), is very satisfactory. The forward-backward asymmetry resulting from reference TDSE calculations can then be compared to the four discriminatory chiral-SFA fingerprints in order to identify the origin of the observed asymmetry. This comparison is performed for each ATI peak, as exemplified in Figs. 6(b), 6(d), 6(f), and 6(h) for the second ATI peak associated to the interaction of the toy-model molecule with the 8- and C-shaped OTC fields, respectively. Contrarily to
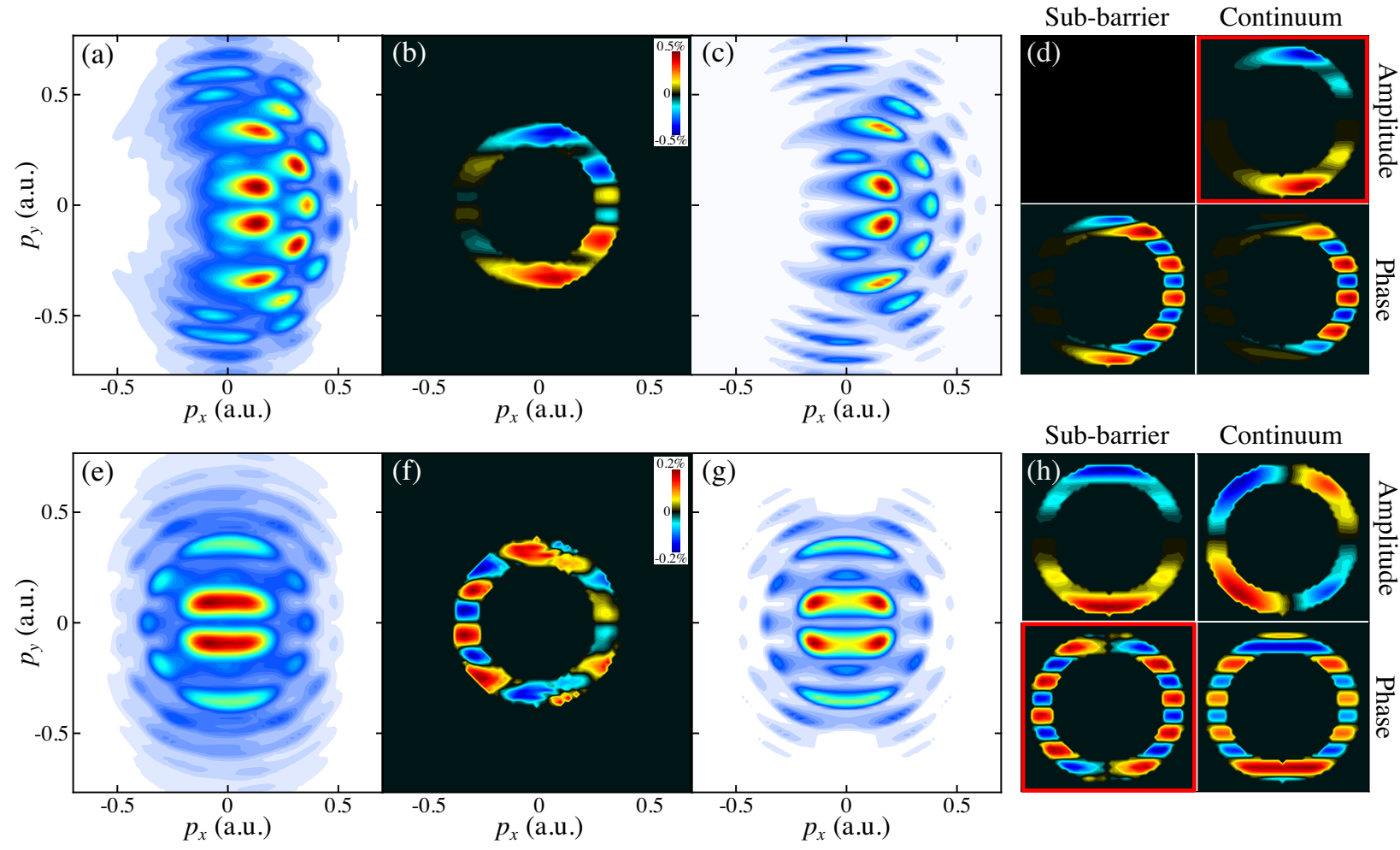

FIG. 6. (a) Projected PAD $\mathcal{P}\left(p_{x}, p_{y}\right)$ obtained by TDSE calculations when the Yukawa-screened toy molecule is ionized by an 8-shaped OTC field with $I_{\omega}=5 \times 10^{13} \mathrm{~W} \cdot \mathrm{cm}^{-2}$ and $r=0.1$. (b) Projected forward-backward asymmetry $\Delta \mathcal{P}^{f / b}\left(p_{x}, p_{y}\right)$ resulting from TDSE calculations, restricted to the second ATI peak and normalized to the maximal value of $\mathcal{P}\left(p_{x}, p_{y}\right)$. (c) SFA counterpart to the TDSE projected PAD. (d) Chiral-SFA asymmetries assuming different sources of chiral response. The TDSE asymmetry in the second ATI signal mainly stems from continuum amplitude effects. (e)-(h) are identical to (a)-(d) but relative to a C-shaped field. The TDSE asymmetry is mainly due to a sub-barrier chiral phase in that case. 
our previous attoclock study, we skip here the analysis of the first ATI peak whose interference patterns are excessively contracted about $\mathbf{p}=\mathbf{0}$ because of the large ponderomotive shift induced by our present driving pulse. A short glance at Figs. 6(b) and 6(d) is enough to observe that, in the case of an 8-shaped field, the TDSE asymmetry is best modeled by a chiral continuum amplitude response. The asymmetry is thus encoded in the TDSE signal by electron scattering in the weak reminiscent long-range chiral potential. Switching from an 8- to C-shaped field, the TDSE asymmetry of Fig. 6(f) exhibits a fringe pattern which is antisymmetric along the second-harmonic $p_{x}$ axis, ruling out the contribution of accumulated continuum phase effects that yield a $p_{x}$-symmetric chiral response. The main origin of the TDSE chiral response thus appears to be in that case a chiral phase acquired in the tunneling process, under the barrier.

These qualitative comparisons reveal the coherent properties of the chiral strong-field interaction. They highlight the interest of subcycle gated interferometry to identify the origin of the chiral response, as a function of the driving field shape. In this respect, the case of C-shaped OTC fields already complements our previous attoclock study: Besides a chiral amplitude effect, tunnel ionization through a chiral barrier also induces an asymmetric phase modulation.

We go a step further by characterizing quantitatively the four contributions to the chiral asymmetry. We focus on the case where a C-shaped OTC field is used, since it provides the most distinctive signatures of the origin (sub-barrier or continuum) and the nature (amplitude or phase) of the process. A first solution could be fitting the TDSE asymmetry as a weighted sum of the four contributions obtained in the chiral SFA and presented in Fig. 6. However, even if the PADs calculated by the two theories resemble each other, the exact position of the interference fringes slightly differs, which makes the direct fitting inappropriate. To circumvent this limitation, we first stay in the chiral-SFA framework. We introduce four functions $\left\{S_{i}(p, \theta, \varphi)\right\}_{i=1, \ldots, 4}$ that fit the four contributions of the computed asymmetry on the basis of the forward $(\theta>0)$ part of the PAD, as a function of the azimuthal angle $\varphi=\tan ^{-1}\left(p_{y} / p_{x}\right)$ for fixed $p$ and $\theta$ :

$$
\begin{gathered}
S_{1}(p, \theta, \varphi)=-P(p,|\theta|, \varphi) \sin \varphi, \\
S_{2}(p, \theta, \varphi)=-\frac{\partial P(p,|\theta|, \varphi)}{\partial \varphi}, \\
S_{3}(p, \theta, \varphi)=-P_{\max }(p,|\theta|)[\sin 2 \varphi+0.2 \sin 4 \varphi], \\
S_{4}(p, \theta, \varphi)=-\operatorname{sgn}(\cos \varphi) \frac{\partial P(p,|\theta|, \varphi)}{\partial \varphi} .
\end{gathered}
$$

Here, $P_{\max }(p,|\theta|)$ is the maximum value of $P(p,|\theta|, \varphi)$ along $\varphi$. The functions $\left\{S_{i}(p, \theta, \varphi)\right\}_{i=1, \ldots, 4}$ capture the main characteristics of the fundamental chiral components. In the case of a sub-barrier amplitude perturbation $\left(S_{1}\right)$, the forward-backward asymmetry can simply be modeled as being proportional to the signal, with an additional sinusoidal modulation as a function of $\varphi$ which reflects the angular dependence of the instantaneous chirality at the time of ionization. Concerning the sub-barrier phase $\left(S_{2}\right)$, a forward-backward asymmetric component $\delta \eta^{f / b}$ in the relative phase between the direct and indirect wave packets leads to an angular streaking shift $\delta \varphi^{f / b}$ of the fringes between the forward and backward signals, as in standard Young's double-slit experiments. The differential shift $\delta \varphi^{f / b}$ is encoded in the derivative of the signal, since $\Delta P^{f / b}(p,|\theta|, \varphi)=P(p,|\theta|, \varphi)-P(p,-|\theta|, \varphi)=$ $P(p,|\theta|, \varphi)-P\left(p,|\theta|, \varphi+\delta \varphi^{f / b}\right) \approx-\delta \varphi^{f / b}[\partial P(p,|\theta|$, $\varphi) / \partial \varphi]$. We observe in Fig. 5(h) that the asymmetry resulting from continuum amplitude effects $\left(S_{3}\right)$ is surprisingly smooth, not showing any interference pattern. This indicates that one of the two families dominates the chiral response, annihilating the contrast of the interference. We indeed checked that the cumulative amplitude chiral perturbation $\alpha$ of Eq. (4) is much larger for indirect pathways than for direct ones, which is intuitively understood through the longer time they spend around the chiral ionic core. Last, continuum phase effects $\left(S_{4}\right)$ lead to a differential forward-backward angular streaking shift of the fringes, similar to the one encoded in $S_{2}$. However, this shift is found in Fig. 6(h) of opposite sign in the $p_{x}>0$ and $p_{x}<0$ hemispheres, which is introduced by $\operatorname{sgn}(\cos \varphi)$ in the expression of $S_{4}$.

Figures 7(a)-7(d) present the reconstructed functions $\left\{S_{i}(p, \theta, \varphi)\right\}$ that reproduce the four contributions of the forward-backward asymmetry computed in the chiral-SFA framework. The reconstruction is illustrated for electrons belonging to the second ATI peak and ejected along $\theta=30^{\circ}$ under the action of a C-shaped field with $I_{\omega}=5 \times 10^{13} \mathrm{~W} \cdot \mathrm{cm}^{-2}$ and $r=0.1$. We further verify that the functions are not linearly dependent, which would prevent using them to decompose the computed total asymmetry. In practice, the $S_{i}$ functions are almost orthogonal, except for the $S_{4}$ one whose normalized overlap with $S_{1}$ is 0.17 .

Next, based on these four functions, we extract the fundamental chiral parameters which define the coherent properties of the sub-barrier and continuum scattering processes. In order to extract these parameters, we turn to the photoelectron angular distribution $P(\mathbf{p})$ and forwardbackward asymmetry $\Delta P^{f / b}(\mathbf{p})$ provided by the TDSE calculations. $P(\mathbf{p})$ is employed to define the basis functions $\left\{S_{i}(\mathbf{p})\right\}$ according to Eqs. (6)-(9). The measurement of chiral effects is then based on the expansion

$$
\Delta P^{f / b}(p, \theta, \varphi)=\sum_{i=1}^{4} a_{i}^{f / b}(p, \theta) S_{i}(p, \theta, \varphi)
$$



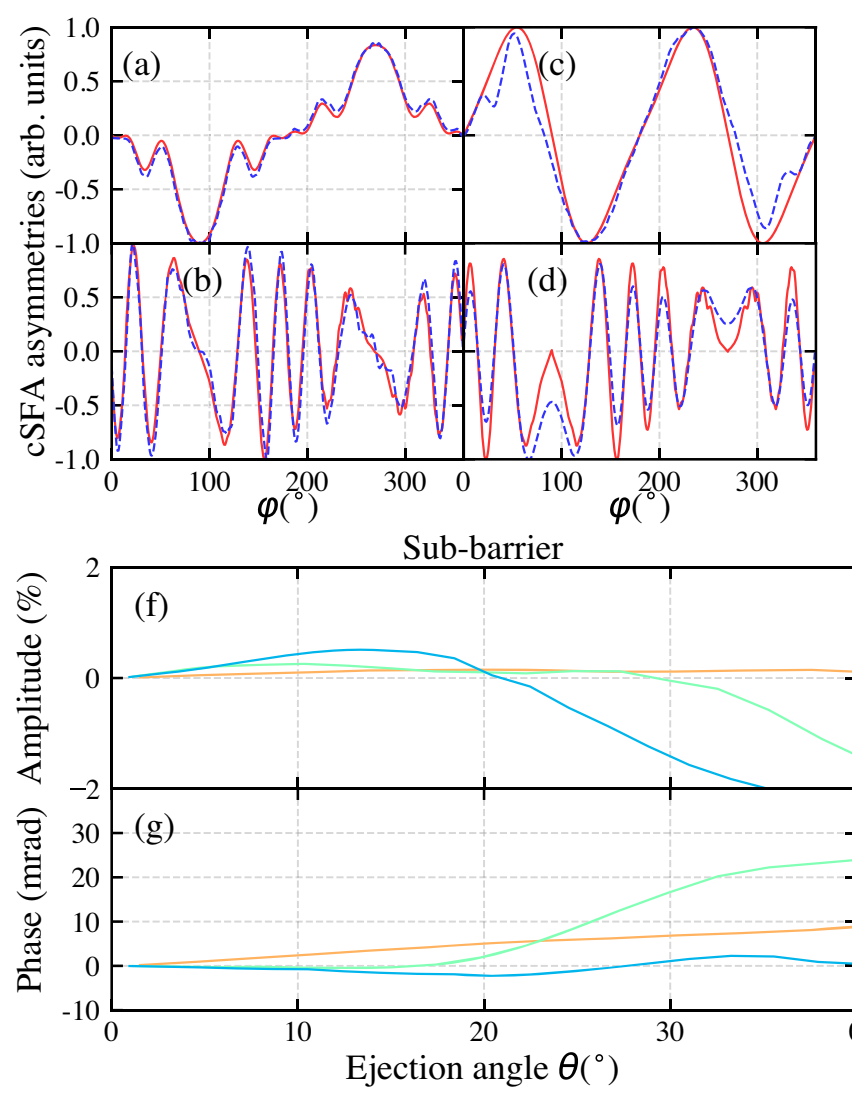

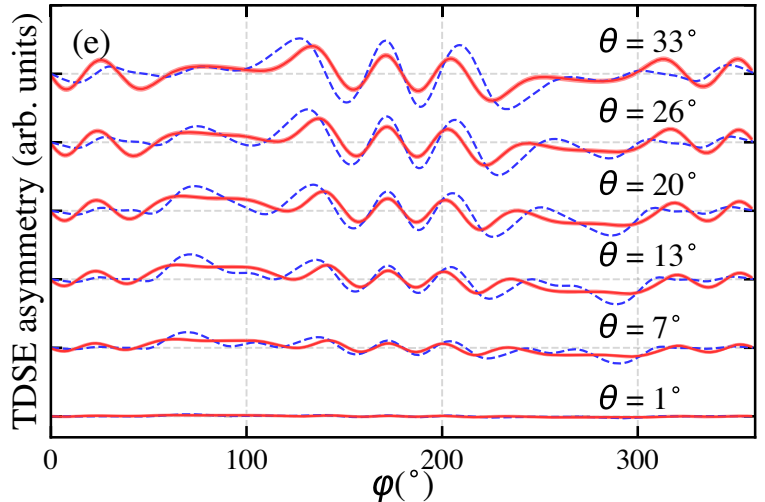

Continuum

(h)

FIG. 7. Extracting the subcycle gated chiral response from TDSE calculations in a Yukawa-screened molecule, driven by an OTC field with $I_{\omega}=5 \times 10^{13} \mathrm{~W} \cdot \mathrm{cm}^{-2}, r=0.1$, and $\phi=0$. (a) Forward-backward asymmetries stemming from instantaneous sub-barrier amplitude effects in the chiral-SFA model (dashed lines) and calculated according to Eq. (6) (continuous lines), as a function of the streaking angle $\varphi$ for ATI2 electrons ejected along $\theta=30^{\circ}$. (b)-(d) The same as (a) but for instantaneous sub-barrier phase, accumulated continuum amplitude, and accumulated continuum phase effects, respectively. (e) $\varphi$ dependence of the forward-backward asymmetry obtained by means of TDSE calculations involving the Yukawa screened potential for ATI2 electrons ejected along selected values of $\theta$ (dashed lines) and associated fits according to Eq. (10) (continuous lines). (f) Chiral sub-barrier amplitude modulations $a_{1}^{f / b}(p, \theta)$ extracted from the fits due to instantaneous tunneling for ATI2-4 electrons (from orange to blue) as functions of $\theta$. (g) Chiral differences $\Delta \eta_{2}^{f / b}(p, \theta)$ in the relative phase between direct and indirect wave packets at the exit of the tunnel. (h) Chiral amplitude modulations $a_{3}^{f / b}(p, \theta)$ accumulated in the continuum. (i) Chiral differences $\Delta \eta_{4}^{f / b}(p, \theta)$ in the relative phase between direct and indirect wave packets accumulated in the continuum.

and consists in determining the coefficients $\left\{a_{i}^{f / b}(p, \theta)\right\}$ for fixed values of $p$ and $\theta$. These coefficients are found by minimizing the difference $\mid \Delta P^{f / b}(p, \theta, \varphi)-$ $\left.\sum_{j} a_{j}^{f / b}(p, \theta) S_{j}(p, \theta, \varphi)\right|^{2}$, using standard least-square fitting algorithms [51]. Figure 7(e) shows comparisons of the reference asymmetry $\Delta P^{f / b}(p, \theta, \varphi)$ and fits [Eq. (10)] for the second ATI peak and a few selected values of $\theta$. The overall agreement is good, confirming that the expansion of the asymmetry $\Delta P^{f / b}$ onto the functions $\left\{S_{i}(\mathbf{p})\right\}$ is reliable to describe the underlying chiral dynamics.

The four coefficients $\left\{a_{j}^{f / b}(p, \theta)\right\}_{j=1, \ldots, 4}$ characterize the contributions of the different sources of asymmetry in the total signal. They are interpreted according to the definition of the basis functions $\left\{S_{j}(\mathbf{p})\right\}_{j=1, \ldots, 4} \cdot a_{1}^{f / b}(p, \theta)$ and $a_{3}^{f / b}(p, \theta)$ are chiral amplitude modulations of the electron yield, since $S_{1}$ and $S_{3}$ are directly proportional to the forward PAD. $S_{2}$ and $S_{4}$ involve the derivative of the PAD so that $a_{2}(p, \theta)$ and $a_{4}(p, \theta)$ are assimilated to chiral angular shifts $\Delta \varphi^{f / b}(p, \theta)$ mentioned above. We refer to the shifts associated to $S_{2}$ and $S_{4}$ as $\Delta \varphi_{2}^{f / b}(p, \theta)$ and $\Delta \varphi_{4}^{f / b}(p, \theta)$, respectively. These angular shifts are converted to chiral differences $\Delta \eta_{2,4}^{f / b}(p, \theta)$ in the relative phase between direct and indirect tunneling wave packets through $\Delta \eta_{2,4}^{f / b}(p, \theta)=2 \pi \Delta \varphi_{2,4}^{f / b}(p, \theta) / i(p, \theta)$, where $i(p, \theta)$ is the angular interfringe of the electron distribution along $\varphi$, for fixed $p$ and $\theta$.

The behaviors of $a_{1}^{f / b}(p, \theta), a_{3}^{f / b}(p, \theta), \Delta \eta_{2}^{f / b}(p, \theta)$, and $\Delta \eta_{4}^{f / b}(p, \theta)$ are presented in Figs. 7(f)-7(i) as a function of the ejection angle $\theta$. For the second ATI peak, the dominant source of asymmetry is a chiral sub-barrier phase 
$\Delta \eta_{2}^{f / b}(p, \theta)$, as already inferred by the visual comparison of Figs. 6(f) and 6(h). As the momentum component of the electron along the light propagation axis increases, the chiral sub-barrier phase increases, reaching approximately $10 \mathrm{mrad}$ at $\theta=40^{\circ}$. The chiral sub-barrier amplitude $a_{1}^{f / b}(p, \theta)$ remains very small (less than $0.2 \%$ ) throughout the entire $\theta$ range. The analysis of the higher-order ATI peaks shows that the main source of asymmetry in the photoelectron angular distribution is always the tunneling process, leading to significant amplitude $a_{1}^{f / b}(p, \theta)$ and phase $\Delta \eta_{2}^{f / b}(p, \theta)$ effects. The magnitude of the chiral subbarrier amplitude increases with the order of the ATI peak, reaching the $1 \%-2 \%$ range at $\theta=40^{\circ}$ for ATI 3 and 4 . The chiral sub-barrier phase reaches more that $25 \mathrm{mrad}$ for ATI3 at $\theta=40^{\circ}$. Regarding continuum dynamics, phase effects $\Delta \eta_{4}^{f / b}(p, \theta)$ remain insignificant. A small chiral continuum amplitude $a_{3}^{f / b}(p, \theta)$ appears for large $\theta$ and high ATI orders, indicating a residual effect of the potential, as already observed with the 8-shaped field in Fig. 6(b). The results in Figs. 7(f) and 7(g) prove that the primary tunneling dynamics are sensitive to chirality. Moreover, it is commonly agreed that in conventional PECD, where the asymmetry is accumulated during continuum electron scattering off the potential, slow electrons are more sensitive to the structure of the molecular potential [5]. Here, we observe an opposite trend-faster electrons show a stronger asymmetry.

\section{DISCUSSION}

The combination of two complementary techniques has enabled us to draw a complete picture of the influence of the chiral molecular potential in strong-field ionization. At long range, the chirality of the potential influences the angular streaking of the electrons in the continuum, inducing a forward-backward asymmetry in the attoclock angles. The chiral potential also induces an asymmetry in the number of electrons ending up in the forward and backward hemispheres. In camphor and fenchone measurements, as well as in toy-model calculations, this asymmetry is larger for higher-energy electrons. This contradicts the usual picture of PECD, in which faster electrons are expected to be less sensitive to the structure of the potential. Furthermore, this asymmetry survives screening of the long-range potential in TDSE calculations. This reflects a different physical origin of the asymmetry, which is not imprinted during scattering in the continuum but under the barrier, during the tunneling process. Subcycle gated chiral interferometry confirms this conclusion by disentangling tunneling and scattering. It shows that chirality induces not only an amplitude modulation, but also a phase asymmetry in the tunnel-ionization dynamics.

The influence of the molecular potential on the tunneling process may not seem surprising. Indeed, it has been long established that the molecular geometry can affect the tunneling probability and the shape of the wave packet at the exit of the tunnel, through the shape of the molecular orbitals [52,53]. Recent works on high-harmonic spectroscopy $[54,55]$ and photoelectron spectroscopy $[26,56]$ also have demonstrated that phase shifts could be imprinted by tunneling. However, two aspects make the current results unique. First, the geometrical factor at play is a 3D symmetry property, which survives molecular orientation averaging. Second, the phase and amplitude modulations observed here do not result solely from the chiral geometry of the potential barrier. They originate from a dynamical phenomenon, imprinted by the interaction of this barrier with a laser field whose polarization direction rotates in time.

The dynamics of electrons under the barrier are a very controversial topic. In our subcycle gated chiral interferometry modeling, we assume that tunneling is instantaneous but sensitive to the optical chirality of light. This is somehow contradictory, since a finite amount of time is required for the light electric field to rotate and, thus, for optical chirality to be expressed. This contradiction can be resolved without introducing a finite tunneling time, by analyzing the process in the complex plane, which is best suited to describe strong-field light-matter interaction $[57,58]$. In this framework, tunneling takes a nonzero imaginary time. The electron follows a complex trajectory under the barrier, where it experiences the influence of both the laser field and the ionic potential [50]. The amplitude and the phase of the electron wave packet released at the exit of the tunnel are thus sensitive to the intrinsic features of both the light and target. This is how the chirality of the latter naturally translates into the observed asymmetry of the tunneling wave packets. Within this chiral tunneling scheme, the higher the kinetic energy of the released electron is, the larger the imaginary time and the tunnel exit distance from the core are. The electron thus follows a longer complex trajectory under the chiral barrier lowered by the chiral field, which is consistent with the larger forward-backward asymmetry observed for high-energy electrons.

The results can also be interpreted using an alternative picture of tunneling dynamics, provided by quantum trajectory analysis of TDSE calculations [59]. Contrarily to noninteracting classical trajectories, quantum trajectories are entangled and can exchange energy. Tunneling thus appears in the quantum trajectory framework in terms of an electron which borrows energy from neighbor trajectories and surmounts the barrier. Here again, the electron can capture asymmetry features in the hopping ionization dynamics associated to tunneling.

In both interpretations of tunneling, the driving field induces electronic ring currents, like in strong-field ionization of atomic targets [60]. The chirality of the molecular potential thus imprints a forward-backward asymmetry on this electron flow, as in the photoexcitation circular 
dichroism mechanism [61], which results in a phase and amplitude asymmetry of the electron wave packet emerging in the continuum.

Our whole investigation is performed in the nonadiabatic tunneling regime, in which electrons get excited before being released in the continuum [33,62]. This excitation could enhance the chiral sensitivity of the process, by creating a chiral bound electron wave packet whose asymmetric features are imprinted in the electrons emerging in the continuum. Nevertheless, we stress that the existence of a sub-barrier phase is established in the pure tunneling regime with $\gamma<1$ [50]. We thus expect that chirosensitive asymmetric patterns should survive in the quasistatic picture of tunnel ionization.

\section{METHODS}

\section{A. Chiral attoclock measurements}

\section{Experimental setup}

The attoclock experiments are performed using the Aurore laser system at CELIA, which delivers up to $7 \mathrm{~mJ}, 26-\mathrm{fs}$ pulses centered around $800 \mathrm{~nm}$, at $1 \mathrm{kHz}$ repetition rate [34]. The beam is split by a 50\%/50\% beam splitter at the entrance of a Mach-Zehnder interferometer. In one of the two arms, we frequency double the infrared pulses using a type-I $200-\mu \mathrm{m}$-thick $\beta$-barium borate (BBO) crystal. Reflections on two dichroic mirrors remove the remaining fundamental wavelength. We place motorized quarter-wave plates in both arms to control independently the polarization state of each color. After the wave plates, all the reflections are at approximately $0^{\circ}$ to avoid introducing polarization state artifacts. A motorized pair of wedges installed in the $800-\mathrm{nm}$ arm allows us to finely control the attosecond delay between the two colors. The two beams are recombined using a dichroic mirror and are focused by an $f=30 \mathrm{~cm}$ lens into the interaction zone of a velocity map imaging spectrometer (VMIS). The VMIS uses an electrostatic lens to project the $3 \mathrm{D}$ photoelectron angular distribution onto a $2 \mathrm{D}$ detector, which is composed of a set of dual microchannel plates imaged by a phosphor screen and a 12-bit CCD camera. The divergence of the $800-\mathrm{nm}$ beam is finely tuned using a telescope in one arm of the Mach-Zehnder to ensure that the 800- and 400-nm beams are focused at the same position after the lens. The solid samples of camphor or liquid fenchone molecules are heated in an oven at $60^{\circ} \mathrm{C}$ and injected in the VMIS through a $250-\mu \mathrm{m}$ nozzle located at approximately $7 \mathrm{~cm}$ from the laser beam.

\section{B. Analysis of experimental photoelectron angular distributions}

The VMIS records the 2D projection of the 3D PAD. When linearly or circularly polarized radiation is used, the 3D PAD $P(\mathbf{p})$ can be retrieved from the 2D projection by Abel inversion. This is not the case with bicircular fields because of the lack of cylindrical symmetry of the interaction. In that case, the 3D PAD can be obtained by using a set of $2 \mathrm{D}$ projections recorded for different orientations [29,35]. To rotate the 3D PAD by an angle $\Phi$ around the laser propagation direction $z$, we scan the delay $\tau$ between the two components of the bicircular field, defined (for left polarization $L$ of the fundamental field) as

$$
\mathbf{E}(t)=\frac{f(t) E_{0}}{\sqrt{2}}(-\{\sin [\omega(t+\tau)]+\sqrt{r} \sin (2 \omega t)\} \hat{\mathbf{x}}+\{\cos [\omega(t+\tau)]+\sqrt{r} \cos (2 \omega t)\} \hat{\mathbf{y}}),
$$

where $f(t)$ is the temporal envelop of the field, $E_{0}$ the peak field value, and $r$ the intensity ratio between the fundamental and second-harmonic components. Figure 8 shows one period of the field obtained with $r=0.1$, for different delays between these two components, corresponding to the delay step used in the experiment $\left(\delta \tau \approx 133\right.$ as, equivalent to a $\delta \Phi \approx 36^{\circ}$ rotation step along $\Phi)$. Each projection of the $3 \mathrm{D}$ PAD is accumulated over $5 \times 10^{4}$ laser shots, and 11 projections are recorded. To improve the tomographic reconstruction, we interpolate the results over a finer $\Phi$ grid with a $1^{\circ}$ step. We then use inverse Radon transform for each slice along $z$ to retrieve the 3D PAD.

The sensitivity of photoelectron spectroscopy to molecular chirality is imprinted as forward-backward asymmetries in the angular distributions of the electrons. This asymmetry, obtained by differentiating the forward
$F(p, \theta, \varphi)=P(p, \theta, \varphi) \quad$ and $\quad$ backward $\quad B(p, \theta, \varphi)=$ $P(p,-\theta, \varphi)$ electron signals (with $\theta>0$ ), reverses sign when switching from one enantiomer to its mirror image. Experimental photoelectron angular distributions are never free from spurious contributions due to experimental artifacts. Thus, photoelectron dichroism experiments always rely on differential measurements to disentangle the genuine chiral signal from asymmetries intrinsic to electron detection artifacts. When using circularly polarized light (CPL), the photoelectron angular distribution has a cylindrical symmetry around the light propagation axis. Switching from one enantiomer to its mirror image is then equivalent to switching the helicity of the ionizing radiation. The normalized forward-backward asymmetry in the electron yield $\Delta P_{\mathrm{CPL}}^{f / b}$ is then equal to the PECD, obtained by differentiating the signals $L$ and $R$ recorded with, respectively, left-handed and right-handed ionizing light: 


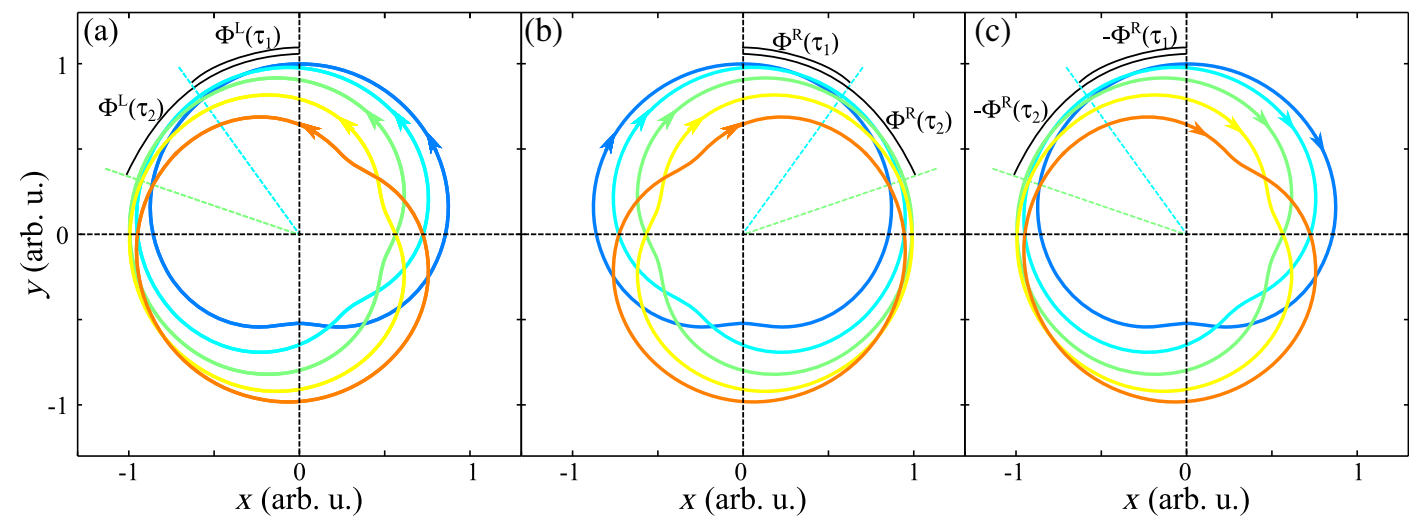

FIG. 8. Evolution of a bicircular field with intensity ratio $r=0.1$ as a function of the delay between the two $\omega-2 \omega$ components, increasing from blue to red by steps of $\delta \tau \approx 133$ as. (a) The fundamental field is left polarized. (b) The fundamental field is right polarized. (c) Alternative geometry for tomographic inversion, switching the sign of the rotation angle $\Phi^{R}$ to obtain the mirror image of the photoelectron angular distribution.

$$
\Delta P_{\mathrm{CPL}}^{f / b}=2 \frac{F-B}{F+B}=2 \frac{L-R}{L+R}=\mathrm{PECD} .
$$

When bicircular fields are used, the equivalence between $\Delta P^{f / b}$ and PECD is lost. Indeed, switching the helicity of the two electric fields induces a mirror imaging of the whole bicircular field (Fig. 8), leading to large angular offsets between the left and right fields. Subtracting the photoelectron angular distributions obtained with opposite bicircular fields, thus, produces a differential distribution, which is largely dominated by this angular offset of the $E$ fields, and

$$
\Delta P_{\text {bircirc }}^{f / b}=2 \frac{F-B}{F+B} \neq 2 \frac{L-R}{L+R} .
$$

How can the chiral response induced by bicircular fields be extracted from a differential measurement? A first solution consists in performing consecutive measurements with a fixed polarization (e.g., $L$ ) in opposite enantiomers $(+)$ and $(-)$, which should show opposite responses, and to average the forward-backward asymmetries:

$$
\Delta P_{L}^{f / b}=\frac{1}{2}\left[\left(F_{L}^{(+)}-B_{L}^{(+)}\right)-\left(F_{L}^{(-)}-B_{L}^{(-)}\right)\right] .
$$

The drawback of this procedure is that it requires switching the enantiomer in the experiment, which takes several tens of minutes, during which the experimental parameters may shift - attosecond stability is indeed necessary to maintain the lab-frame orientation of the bicircular field. This issue can be circumvented by taking a closer look at the effect of the helicity of the bicircular field in the experiment.

Switching the helicities of the fundamental and secondharmonic field induces a mirror symmetry on the bicircular field. This operation also reverses the scanning direction of the projection angle with two-color delay: $\Phi^{L}(\tau)=$ $-\Phi^{R}(\tau)$ [Fig. 8(b)]. If we ignore this angular scanning direction reversal and perform the tomographic reconstruction defining the same projection angles for the $R$ polarization than for the $L$ one [Fig. 8(c)], we obtain the reconstructed distribution $P_{R}^{*( \pm)}$ which is the mirror image of the actual distribution $P_{R}^{( \pm)}$. For symmetry reasons, this distribution is the same as the one obtained with the opposite helicity in the opposite enantiomer: $P_{R}^{*( \pm)}=P_{L}^{(\mp)}$. The chiral response can, thus, be extracted from differential measurements using a single enantiomer by

$$
\begin{aligned}
\Delta P_{L}^{f / b} & =\frac{1}{2}\left[\left(F_{L}^{(+)}-B_{L}^{(+)}\right)-\left(F_{R}^{*(+)}-B_{R}^{*(+)}\right)\right] \\
& =\frac{1}{2}\left[\left(F_{L}^{(+)}-B_{L}^{(+)}\right)-\left(F_{L}^{(-)}-B_{L}^{(-)}\right)\right] .
\end{aligned}
$$

The validity of this equation is illustrated in Fig. 9, which shows the forward-backward asymmetry obtained by applying the two methods to measurements in camphor, using a bicircular field with total intensity $I=8 \times 10^{12} \mathrm{~W} \cdot \mathrm{cm}^{-2}$ and $r=I_{2 \omega} / I_{\omega}=1$ between the two frequency components.

The differential measurement of the forward-backward asymmetry enables us to isolate the chiral response from experimental artifacts. The artifact-free 3D PAD can be obtained by summing $\Delta P_{L}^{f / b}$ with the forward-backwardsymmetrized 3D PAD:

$$
\begin{aligned}
P_{L}= & \frac{1}{4}\left[\left(F_{L}^{(+)}-B_{L}^{(+)}\right)-\left(F_{R}^{*(+)}-B_{R}^{*(+)}\right)\right] \\
& +\frac{1}{4}\left[\left(F_{L}^{(+)}+B_{L}^{(+)}\right)+\left(F_{R}^{*(+)}+B_{R}^{*(+)}\right)\right] .
\end{aligned}
$$

\section{Mirroring of attoclock measurements}

In order to verify our attoclock analysis of the signal, we compare measurements in opposite enantiomers of camphor molecules (Fig. 10). These measurements are 
$\frac{1}{2}\left[\left(F_{L}^{(+)}-B_{L}^{(+)}\right)-\left(F_{L}^{(-)}-B_{L}^{-)}\right)\right]$

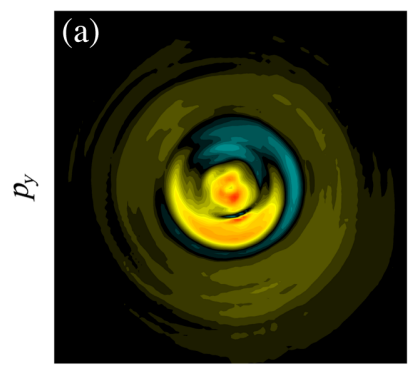

$p_{x}$

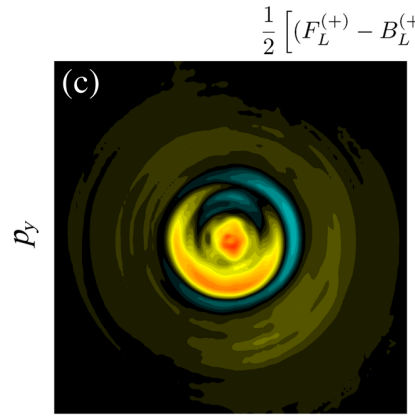

$p_{X}$

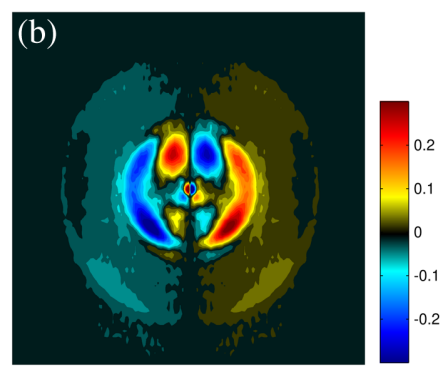

$p_{z}$

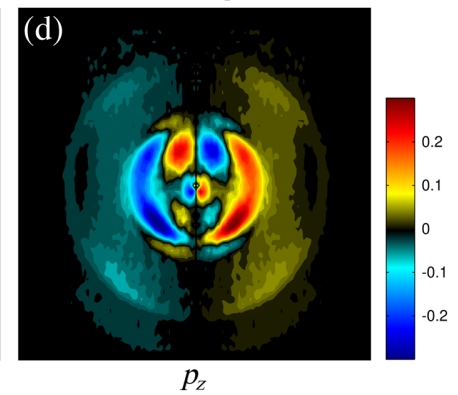

FIG. 9. Comparison of the two differential measurements in camphor molecules subject to a bicircular field with $I=$ $8 \times 10^{12} \mathrm{~W} \cdot \mathrm{cm}^{-2}$ and $r=I_{2 \omega} / I_{\omega}=1$. (a) Chiral response in the forward hemisphere projected in the laser polarization plane and (b) chiral response projected in the $(y, z)$ plane, obtained by differential measurements on opposite enantiomers at fixed polarization. (c),(d) The same as (a),(b) but obtained by differential measurements with opposite field polarizations for a given enantiomer.

conducted at lower intensity $I=8 \times 10^{12} \mathrm{~W} \cdot \mathrm{cm}^{-2}$ and using a different ratio between the fundamental and second-harmonic fields, $r=1$. The evolution of the streaking angle with electron ejection direction is clearly different from that observed in the strong-field regime. The two ATI peaks show the same behavior, with a $\Delta \varphi_{0}^{f / b}$ reaching $5^{\circ}$ and a relative asymmetry in the electron yield reaching $-2 \%$ at $\theta=40^{\circ}$. Switching from (+)- to $(-)$-camphor reverses the sign of these two signals, confirming that they are genuine chiroptical observables.

\section{Experimental details on chiral photoionization by OTC fields}

For this experiment, we use the $50-\mathrm{W}$ laser beam provided by the BlastBeat laser system at CELIA (Tangerine SP, Amplitude Systemes), delivering 135-fs pulses at $1030-\mathrm{nm}$ wavelength with $166-\mathrm{kHz}$ repetition rate. The beam is split in a Mach-Zehnder interferometer, one arm remaining at the fundamental frequency and the other arm being used to perform second-harmonic generation into a 1-mm-thick type-I BBO crystal. The delay between the two arms is coarsely tuned with a pair of mirrors mounted on a manual translation stage and actively stabilized using a piezointertially controlled mirror mount. The beams are recombined collinearly by a dichroic mirror and sent through a calcite plate, which ensures that their polarizations are perfectly orthogonal. The relative phase between the fundamental and second-harmonic beam is finely scanned using a pair of fused silica wedges placed after the calcite plate. The two collinear beams are focused by an $f=60 \mathrm{~cm}$ lens into the interaction region of a COLTRIMS. A slightly detuned telescope is placed in the $\omega$ arm to compensate the chromatic aberration of the lenses so that the foci of the $\omega$ and $2 \omega$ beams overlap at the center of the COLTRIMS. The (+)-fenchone molecules are carried by two bars of $\mathrm{N}_{2}$ through a $30-\mu \mathrm{m}$ nozzle coupled to a $200-\mu \mathrm{m}$ skimmer. The ionized electrons are accelerated by an electric field and guided by a magnetic field toward a
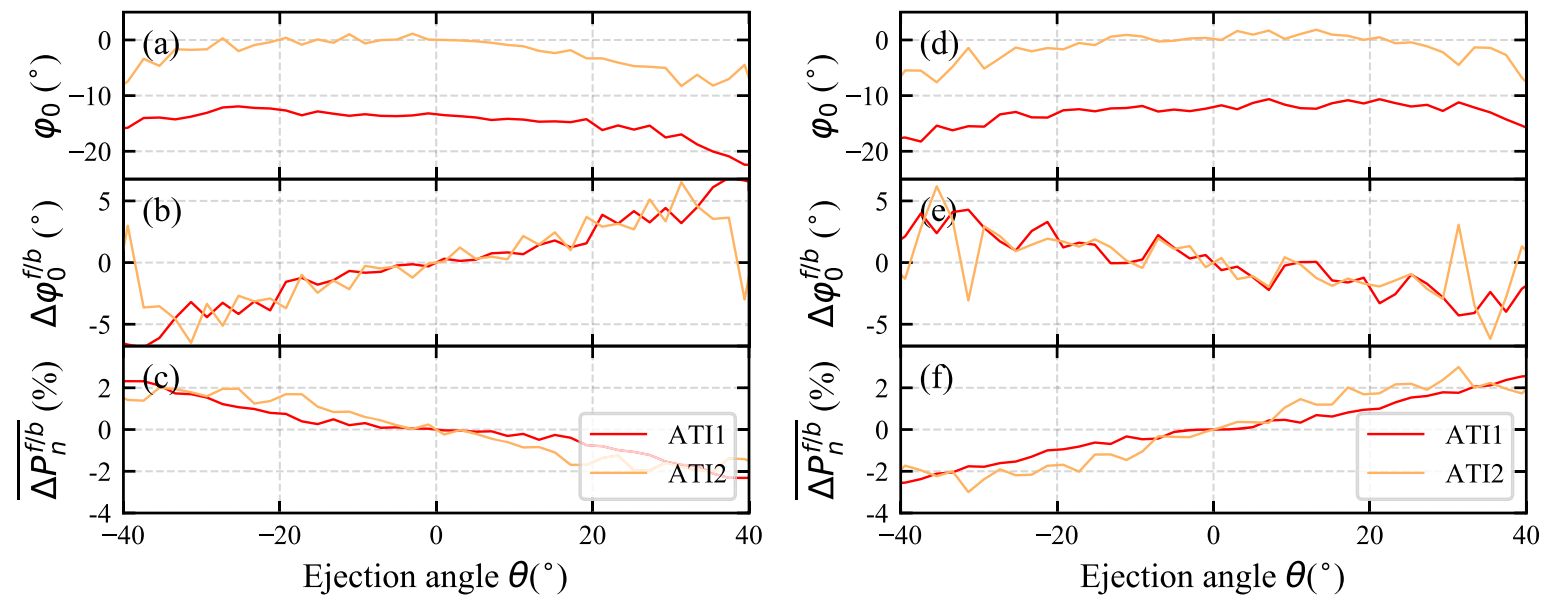

FIG. 10. Enantiosensitivity of attoclock measurements in camphor at $I=8 \times 10^{12} \mathrm{~W} . \mathrm{cm}^{-2}$ with $r=1$. (a) Attoclock streaking angle $\varphi_{0}$ of the first two ATI peaks as a function of the electron ejection angle $\theta$ in (+)-camphor. (b) Forward-backward asymmetry in the attoclock streaking angles $\Delta \varphi_{0}^{f / b}(\theta)$. (c) Forward-backward asymmetry in the intensity of the ATI peaks, $\overline{\Delta P_{n}^{f / b}}(\theta)$. (d)-(f) are identical to (a)-(c) but relative to (-)-camphor. 
set of microchannel plates. Their position and arrival time are measured by delay-line anodes, to finally record the 3D momentum distribution of the photoelectrons. For each relative phase between the two components of the OTC field, the $3 \mathrm{D}$ electron distribution is accumulated until $10^{8}$ electrons are detected.

The absolute phase between the $\omega$ and $2 \omega$ fields is determined by analyzing the evolution of the photoelectron momentum distribution in the laser polarization plane. This leads us to conclude that the phases reported in Ref. [15], in which the 3D distributions are not measured, are offset by $\pi / 2$. This means that the ionization probability is not maximized when the laser field is maximum $(\phi=0 \pm \pi)$ as we assumed in Ref. [15]. Our theoretical calculations confirm this behavior.

\section{E. The toy-model molecule and TDSE calculations}

We employ the same toy-model molecule and TDSE framework as in the ESCARGOT study [15]. Therefore, we only outline our approach here and then focus on the convergence of the chiral attoclock calculations.

\section{General framework}

The fictitious molecule consists of a single electron evolving in the field of four nuclei with charges $Z_{1}=-1.9$ and $Z_{2-4}=0.9$, respectively, frozen throughout the interaction at $\mathbf{R}_{\mathbf{1}}=\mathbf{0}, \mathbf{R}_{\mathbf{2}}=\hat{\mathbf{x}}, \mathbf{R}_{\mathbf{3}}=2 \hat{\mathbf{y}}$, and $\mathbf{R}_{\mathbf{4}}=3 \hat{\mathbf{z}}$. The bound and continuum states $\phi_{\epsilon_{i}}$ of the molecule are obtained by diagonalizing the field-free Hamiltonian $H_{0}=$ $-\frac{1}{2} \nabla^{2}+V_{\text {mol }}(\mathbf{r})=-\frac{1}{2} \nabla^{2}-\sum_{i=1}^{4} Z_{i} /\left|\mathbf{r}-\mathbf{R}_{\mathbf{i}}\right|$ in a basis of primitive functions $\chi_{i l_{i} m_{i}}(\mathbf{r})=j_{l_{i}}\left(k_{i} r\right) \mathcal{Y}_{l_{i} m_{i}}^{\text {cos, sin }}\left(\Omega_{\mathbf{r}}\right)$ whose radial parts $j_{l_{i}}\left(k_{i} r\right)$ are spherical Bessel functions, while $\mathcal{Y}_{l_{i} m_{i}}^{\text {cossin }}\left(\Omega_{\mathbf{r}}\right)$ are real spherical harmonics with $0 \leq l_{i} \leq l_{\max }$ and $0 \leq m_{i} \leq l_{i}$. The electron motion is confined within a spherical box of radius $r_{\text {max }}$ so that we introduce only the $\chi_{i l_{i} m_{i}}(\mathbf{r})$ functions with $k_{i}$ values such that $j_{l_{i}}\left(k_{i} r_{\max }\right)=0$. Electron momenta $k_{i}$ are further restricted to $0 \leq k_{i} \leq k_{\max }$. Here, we employ $r_{\max }=200$ a.u., $k_{\max }=7$ a.u., and $l_{\max }=14$, which guarantees the convergence of the ESCARGOT results as in Ref. [15]. Among the diagonalized states such that $\phi_{\epsilon_{j}}(\mathbf{r})=\sum_{i, l_{i}, m_{i}} D_{\epsilon_{j}, i l_{i} m_{i}} \chi_{i l_{i} m_{i}}(\mathbf{r})$, the fundamental one has energy $\epsilon_{0}=-9 \mathrm{eV}$.

For a given molecular orientation $\hat{\mathbf{R}}$, the TDSE is solved in the velocity gauge according to

$$
\left(H_{0}-i \mathcal{R}(\hat{\mathbf{R}})[\mathbf{A}(t)] \cdot \boldsymbol{\nabla}-i \frac{\partial}{\partial t}\right) \Phi(\hat{\mathbf{R}}, \mathbf{r}, t)=0,
$$

where $\mathcal{R}(\hat{\mathbf{R}})[\mathbf{A}(t)]$ is the vector potential associated to the driving field passively rotated from the laboratory to the molecular frame by means of the rotation matrix $\mathcal{R}(\hat{\mathbf{R}})$. In practice, we introduce a flat-shape four-cycle pulse with $\lambda=800 \mathrm{~nm}$, including one $\omega$ cycle ascending and descending ramps, in both attoclock and ESCARGOT calculations. $\Phi(\hat{\mathbf{R}}, \mathbf{r}, t)$ is the total wave function which is expanded onto the $H_{0}$ eigenstates with energies $\epsilon_{j} \leq 2$ a.u. as $\Phi(\hat{\mathbf{R}}, \mathbf{r}, t)=\sum_{j} a_{j}(\hat{\mathbf{R}}, t) \phi_{\epsilon_{j}}(\mathbf{r}) e^{-i \epsilon_{j} t}$. While $a_{j}(\hat{\mathbf{R}}$, $t=0)=\delta_{j 0}$, the ionizing part of the total wave function is extracted at the end of the interaction $(t=\tau)$ as

$$
\Phi^{\mathrm{ion}}(\hat{\mathbf{R}}, \mathbf{r}, \tau)=\sum_{j / \epsilon_{j}>0} a_{j}(\hat{\mathbf{R}}, \tau) \phi_{\epsilon_{j}}(\mathbf{r}) e^{-i \epsilon_{j} \tau} .
$$

It can be alternatively written as

$\Phi^{\mathrm{ion}}(\hat{\mathbf{R}}, \mathbf{r}, \tau)=\sum_{i, l_{i}, m_{i}} b_{i l_{i} m_{i}}(\hat{\mathbf{R}}, \tau) j_{l_{i}}\left(k_{i} r\right) \mathcal{Y}_{l_{i} m_{i}}^{\cos , \sin }\left(\Omega_{\mathbf{r}}\right)$,

where $b_{i l_{i} m_{i}}(\hat{\mathbf{R}}, \tau)=\sum_{l_{j}, m_{j}, n / \epsilon_{n}>0} a_{n}(\hat{\mathbf{R}}, \tau) e^{-i \epsilon_{n} \tau} D_{\epsilon_{n}, j l_{j} m_{j}} \delta_{l_{i} l_{j}}$ $\delta_{m_{i} m_{j}}$. In the lab frame, one thus has

$\Phi_{\mathrm{lab}}^{\mathrm{ion}}(\hat{\mathbf{R}}, \mathbf{r}, \tau)=\sum_{i, l_{i}, m_{i}} b_{i l_{i} m_{i}}(\hat{\mathbf{R}}, \tau) j_{l_{i}}\left(k_{i} r\right) \mathcal{R}^{-1}(\hat{\mathbf{R}})\left[\mathcal{Y}_{l_{i} m_{i}}^{\cos , \sin }\left(\Omega_{\mathbf{r}}\right)\right]$

or, equivalently in momentum space,

$\Phi_{\mathrm{lab}}^{\mathrm{ion}}(\hat{\mathbf{R}}, \mathbf{p}, \tau)=\sum_{i, l_{i}, m_{i}} b_{i l_{i} m_{i}}(\hat{\mathbf{R}}, \tau) \tilde{j}_{i l_{i}}(p) \mathcal{R}^{-1}(\hat{\mathbf{R}})\left[\mathcal{Y}_{l_{i} m_{i}}^{\cos , \sin }\left(\Omega_{\mathbf{p}}\right)\right]$,

where $\tilde{j}_{i i_{i}}(p)$ is the radial part of the momentum wave function associated to the primitive $\chi_{i l_{i} m_{i}}(\mathbf{r})$ function.

We obtain the momentum density of ionized electrons from a sample of randomly oriented molecules at the end of the interaction as

$$
P(\mathbf{p})=\frac{1}{8 \pi^{2}} \int d \hat{\mathbf{R}}\left|\Phi_{\mathrm{lab}}^{\mathrm{ion}}(\hat{\mathbf{R}}, \mathbf{p}, \tau)\right|^{2} .
$$

Molecular orientations $\hat{\mathbf{R}}$ are defined in terms of $(\alpha, \beta, \gamma)$ Euler angles [63], and the integral (22) is evaluated as a numerical quadrature over countable orientations with angular spacings $\Delta \alpha=\Delta \beta=\Delta \gamma=\pi / 5 \mathrm{rad}$.

All this theoretical framework remains identical in the case of calculations involving a Yukawa-type long-range cutoff of the molecular potential, which is then defined as $V_{\text {mol }}(\mathbf{r}) \equiv V_{\text {mol }}(r, \theta, \varphi)=-\sum_{i=1} 4 Z_{i} /\left|\mathbf{r}-\mathbf{R}_{\mathbf{i}}\right|$ for $r \leq r_{0}$, while $V_{\text {mol }}(r, \theta, \varphi)=-e^{-\left(r-r_{0}\right)} \sum_{i=1} 4 Z_{i} /\left|\mathbf{r}-\mathbf{R}_{\mathbf{i}}\right|$ for $r \geq r_{0}$. We do not change the nuclear charges to leave the inner part of the molecular potential unchanged, but the Yukawa term makes the ionization potential of the screened system slightly smaller: $I_{P}=-\epsilon_{0}=7.2 \mathrm{eV}$. This has no significant consequences on the ionization regime experienced by the molecule, since the Keldysh parameter varies from 1.23 in the unscreened case to 1.03 in the Yukawa one 
for an orthogonally polarized two-color field defined by $I_{\omega}=5 \times 10^{13} \mathrm{~W} \cdot \mathrm{cm}^{-2}$ and $r=0.1$.

\section{Convergence of the attochiral calculations}

The convergence of ESCARGOT calculations is presented in Ref. [15]. We focus in this section on the reason why the intensity $I_{\omega}$ of the fundamental component of the bicircular field is restricted to $2 \times 10^{13} \mathrm{~W} \cdot \mathrm{cm}^{-2}$ in the attoclock calculations.

The reliability of our TDSE description depends on the underlying parameters $r_{\max }, k_{\max }$, and $l_{\max } \cdot r_{\max }$ characterizes the range of the spherical box within which the electron dynamics can be fairly described. Too small values of $r_{\max }$ lead to PAD and related asymmetry patterns blurred by ingoing waves associated to reflections of the ionizing electron flux onto the wall of the confinement box. This indeed happens in the high-energy tail of the PAD, which is not of interest in the present work. It is clear from Figs. 4 and 6 that $r_{\max }=200$ a.u. is large enough to obtain the four first ATI peaks free from reflection blurring for the rather short pulses employed in both attoclock and ESCARGOT calculations. $k_{\max }$ is the largest electron wave vector introduced in our basis of primitive Bessel functions. It has a large value, $k_{\max }=7$ a.u., which is necessary to describe accurately the lowest-lying bound eigenstates of the field-free Hamiltonian in terms of oscillating Bessel functions [64-66]. The diagonalization of the Hamiltonian yields eigenstates $\phi_{\epsilon_{j}}(\mathbf{r})$ with energy up to $\epsilon_{j}=k_{\max }^{2} / 2$, which is more than necessary to describe the strong-field-induced ionization processes of present interest - in practice, only eigenstates with energy $\epsilon_{j} \leq 2$ a.u. are introduced in the spectral TDSE expansion.

$l_{\max }$ is the largest angular momentum involved in the single-center partial-wave decomposition (19) of the total wave function. This is the most constraining parameter in our TDSE calculations. In the strong-field ionization regime, the electron indeed gains considerable kinetic momentum through ionization, and $l_{\max }$ increases nonlinearly as the intensity of the driving field increases. On the other hand, the number of primitive functions $\chi_{i l_{i} m_{i}}(\mathbf{r})$ employed in our spectral TDSE scheme scales as $N=\left(r_{\max } / \pi\right) k_{\max }\left(l_{\max }+1\right)^{2}$. The diagonalization operation count is $N^{3}$ (which scales as $l_{\max }^{6}$ ), and the time taken to integrate TDSE is proportional to $N^{2} \sim l_{\max }^{4}$. Therefore, spectral TDSE computations become prohibitively cumbersome as $l_{\max }$ increases. In practice, we set $l_{\max }=14$. To rationalize the convergence of our calculations with respect to $l_{\max }$, we compute the contribution of the angular momentum $l$ to the ionizing density at the end of the interaction as

$$
C_{l}=\frac{1}{8 \pi^{2} \mathcal{N}} \sum_{i, l_{i}, m_{i}} \delta_{l l_{i}} \int d \hat{\mathbf{R}}\left|b_{i l_{i} m_{i}}(\hat{\mathbf{R}}, \tau)\right|^{2} \int_{0}^{r_{\max }} d r r^{2} j_{l_{i}}^{2}\left(k_{i} r\right),
$$

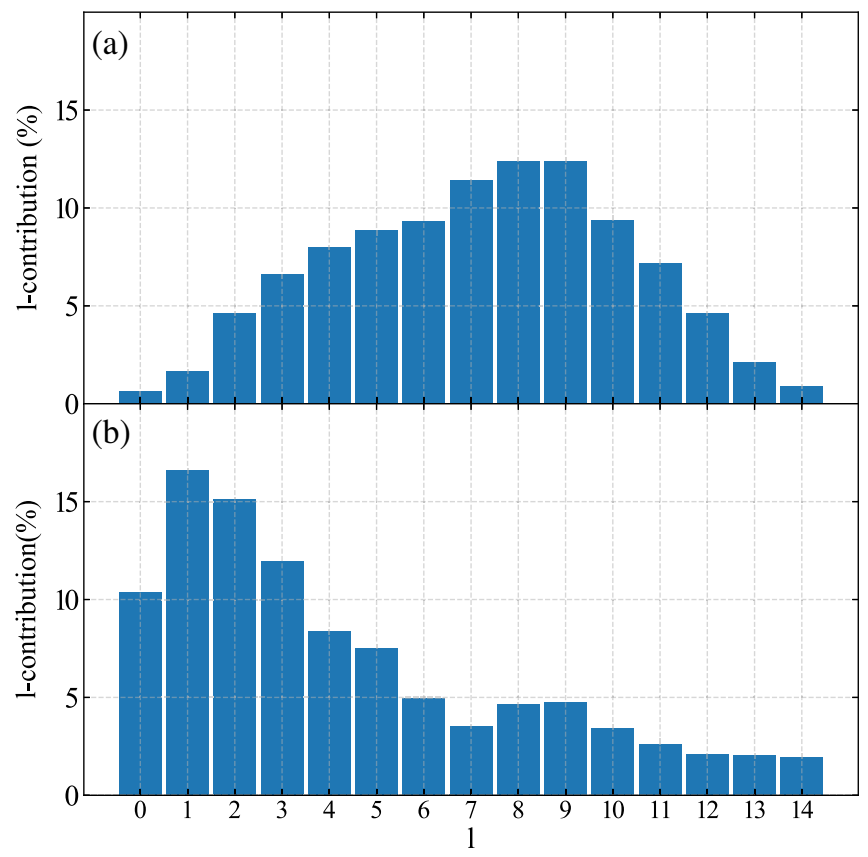

FIG. 11. Convergence of TDSE calculations with respect to the highest angular momentum $l$ introduced in the computations: $C_{l}$ contributions to the ionizing density when the Yukawa-screened toy molecule is subject to a bicircular corotating field with intensity $I_{\omega}=2 \times 10^{13} \mathrm{~W} . \mathrm{cm}^{-2}$ and $r=0.1$ (a) and to an OTC field with $I_{\omega}=5 \times 10^{13}$ W.cm ${ }^{-2}$ and $r=0.1$ (b).

where $\mathcal{N}=\left(1 / 8 \pi^{2}\right) \int d \hat{\mathbf{R}}<\Phi_{\text {lab }}^{\text {ion }}(\hat{\mathbf{R}}, \mathbf{r}, \tau) \mid \Phi_{\text {lab }}^{\text {ion }}(\hat{\mathbf{R}}, \mathbf{r}, \tau)>_{\mathbf{r}}$. The contributions $C_{l}$ are presented in Fig. 11 for the Yukawa-screened attoclock and ESCARGOT calculations presented in Figs. 4 and 6, respectively. Even if $C_{l_{\max }=14}$ does not vanish exactly, the behavior of $C_{l}$ as a function of $l$ indicates a satisfactory convergence. It can be noted that $C_{l}$ peaks about a higher angular momentum when the molecule is submitted to bicircular corotating fields, even if the OTC field introduced in the ESCARGOT calculations has a higher intensity. This stems from the dipolar selection rules inherent to the absorption of circularly and linearly polarized photons, which induce a propensity to populate higher $(l, m)$-partial waves in the former case. This propensity increases as $I_{\omega}$ increases, so that a fair description of ionization by a bicircular field with $I_{\omega}>2 \times 10^{13} \mathrm{~W} \cdot \mathrm{cm}^{-2}$ requires $l_{\max }>14$. Since we set $l_{\max }$ to 14 to reduce the computational cost, we limit our attoclock calculations to $I_{\omega}=2 \times 10^{13} \mathrm{~W} \cdot \mathrm{cm}^{-2}$.

\section{ACKNOWLEDGMENTS}

We thank R. Bouillaud, N. Fedorov, and L. Merzeau for technical assistance. This project has received funding from the European Research Council (ERC) under the European Union's Horizon 2020 research and innovation program No. 682978-EXCITERS, 864127-ATTOGRAM, and 654148-Laserlab-Europe. We acknowledge the financial 
support of the Région Nouvelle Aquitaine (RECHIRAM). N.D. acknowledges support of the Robin Chemers Neustein Professorial Chair. N. D. acknowledges the Minerva Foundation, the Israeli Science Foundation. S. Beaulieu acknowledges the support of a NSERC Vanier Canada Graduate Scholarship.

E. B., S. L., and S. R. contributed equally to this work.

[1] B. Ritchie, Theory of the Angular Distribution of Photoelectrons Ejected from Optically Active Molecules and Molecular Negative Ions, Phys. Rev. A 13, 1411 (1976).

[2] N. Böwering, T. Lischke, B. Schmidtke, N. Müller, T. Khalil, and U. Heinzmann, Asymmetry in Photoelectron Emission from Chiral Molecules Induced by Circularly Polarized Light, Phys. Rev. Lett. 86, 1187 (2001).

[3] C. Lux, M. Wollenhaupt, T. Bolze, Q. Liang, J. Köhler, C. Sarpe, and T. Baumert, Circular Dichroism in the Photoelectron Angular Distributions of Camphor and Fenchone from Multiphoton Ionization with Femtosecond Laser Pulses, Angew. Chem., Int. Ed. 51, 5001 (2012).

[4] C. S. Lehmann, N. B. Ram, I. Powis, and M. H. M. Janssen, Imaging Photoelectron Circular Dichroism of Chiral Molecules by Femtosecond Multiphoton Coincidence Detection, J. Chem. Phys. 139, 234307 (2013).

[5] S. Beaulieu, A. Ferré, R. Géneaux, R. Canonge, D. Descamps, B. Fabre, N. Fedorov, F. Légaré, S. Petit, T. Ruchon, V Blanchet, Y. Mairesse, and B. Pons, Universality of Photoelectron Circular Dichroism in the Photoionization of Chiral Molecules, New J. Phys. 18, 102002 (2016).

[6] A. D. Müller, E. Kutscher, A. N. Artemyev, and P. V. Demekhin, Photoelectron Circular Dichroism in the Multiphoton Ionization by Short Laser Pulses: III. Photoionization of Fenchone in Different Regimes, J. Chem. Phys. 152, 044302 (2020).

[7] D. Patterson and M. Schnell, New Studies on Molecular Chirality in the Gas Phase: Enantiomer Differentiation and Determination of Enantiomeric Excess, Phys. Chem. Chem. Phys. 16, 11114 (2014).

[8] M. H. M. Janssen and I. Powis, Detecting Chirality in Molecules by Imaging Photoelectron Circular Dichroism, Phys. Chem. Chem. Phys. 16, 856 (2014).

[9] L. Nahon, G. A. Garcia, and I. Powis, Valence Shell One-Photon Photoelectron Circular Dichroism in Chiral Systems, J. Electron Spectrosc. Relat. Phenom. 204, 322 (2015).

[10] G. A. Garcia, L. Nahon, S. Daly, and I. Powis, Vibrationally Induced Inversion of Photoelectron Forward-Backward Asymmetry in Chiral Molecule Photoionization by Circularly Polarized Light, Nat. Commun. 4, 2132 (2013).

[11] A. Comby, S. Beaulieu, M. Boggio-Pasqua, D. Descamps, F. Légaré, L. Nahon, S. Petit, B. Pons, B. Fabre, Y. Mairesse, and V. Blanchet, Relaxation Dynamics in Photoexcited Chiral Molecules Studied by Time-Resolved Photoelectron Circular Dichroism: Toward Chiral Femtochemistry, J. Phys. Chem. Lett. 7, 4514 (2016).

[12] S. Beaulieu, A. Comby, B. Fabre, D. Descamps, A. Ferré, G. Garcia, R. Géneaux, F. Légaré, L. Nahon, S. Petit,
T. Ruchon, B. Pons, V. Blanchet, and Y. Mairesse, Probing Ultrafast Dynamics of Chiral Molecules Using TimeResolved Photoelectron Circular Dichroism, Faraday Discuss. 194, 325 (2016).

[13] I. Powis, in Advances in Chemical Physics, edited by S. A. Rice (Wiley, New York, 2008), pp. 267-329.

[14] S. Beaulieu, A. Comby, A. Clergerie, J. Caillat, D. Descamps, N. Dudovich, B. Fabre, R. Géneaux, F. Légaré, S. Petit, B. Pons, G. Porat, T. Ruchon, R. Taïeb, V. Blanchet, and Y. Mairesse, Attosecond-Resolved Photoionization of Chiral Molecules, Science 358, 1288 (2017).

[15] S. Rozen, A. Comby, E. Bloch, S. Beauvarlet, D. Descamps, B. Fabre, S. Petit, V. Blanchet, B. Pons, N. Dudovich, and Y. Mairesse, Controlling Subcycle Optical Chirality in the Photoionization of Chiral Molecules, Phys. Rev. X 9, 031004 (2019).

[16] I. Powis, C. J. Harding, G. A. Garcia, and L. Nahon, A Valence Photoelectron Imaging Investigation of Chiral Asymmetry in the Photoionization of Fenchone and Camphor, Chem. Phys. Chem. 9, 475 (2008).

[17] G. Hartmann, M. Ilchen, P. Schmidt, C. Küstner-Wetekam, C. Ozga, F. Scholz, J. Buck, F. Trinter, J. Viefhaus, A. Ehresmann, M. S. Schöffler, A. Knie, and P. V. Demekhin, Recovery of High-Energy Photoelectron Circular Dichroism through Fano Interference, Phys. Rev. Lett. 123, 043202 (2019).

[18] M. Lewenstein, P. Balcou, M. Y. Ivanov, A. L'Huillier, and P. B. Corkum, Theory of High-Harmonic Generation by Low-Frequency Laser Fields, Phys. Rev. A 49, 2117 (1994).

[19] I. Dreissigacker and M. Lein, Photoelectron Circular Dichroism of Chiral Molecules Studied with a Continuum-State-Corrected Strong-Field Approximation, Phys. Rev. A 89, 053406 (2014).

[20] K. Fehre, S. Eckart, M. Kunitski, C. Janke, D. Trabert, J. Rist, M. Weller, A. Hartung, M. Pitzer, L. P. H. Schmidt, T. Jahnke, R. Dörner, and M. S. Schöffler, Angular Streaking in Strong Field Ionization of Chiral Molecules, Phys. Rev. Research 1, 033045 (2019).

[21] P. Eckle, M. Smolarski, P. Schlup, J. Biegert, A. Staudte, M. Schöffler, H. G. Muller, R. Dörner, and U. Keller, Attosecond Angular Streaking, Nat. Phys. 4, 565 (2008).

[22] A. S. Landsman, M. Weger, J. Maurer, R. Boge, A. Ludwig, S. Heuser, C. Cirelli, L. Gallmann, and U. Keller, Ultrafast Resolution of Tunneling Delay Time, Optica 1, 343 (2014).

[23] L. Torlina, F. Morales, J. Kaushal, I. Ivanov, A. Kheifets, A. Zielinski, A. Scrinzi, H. G. Muller, S. Sukiasyan, M. Ivanov, and O. Smirnova, Interpreting Attoclock Measurements of Tunnelling Times, Nat. Phys. 11, 503 (2015).

[24] U. S. Sainadh, H. Xu, X. Wang, A. Atia-Tul-Noor, W. C. Wallace, N. Douguet, A. Bray, I. Ivanov, K. Bartschat, A. Kheifets, R. T. Sang, and I. V. Litvinyuk, Attosecond Angular Streaking and Tunnelling Time in Atomic Hydrogen, Nature (London) 568, 75 (2019).

[25] M. Richter, M. Kunitski, M. Schöffler, T. Jahnke, L. P. H. Schmidt, M. Li, Y. Liu, and R. Dörner, Streaking Temporal Double-Slit Interference by an Orthogonal Two-Color Laser Field, Phys. Rev. Lett. 114, 143001 (2015).

[26] M. Han, P. Ge, Y. Shao, M.-M. Liu, Y. Deng, C. Wu, Q. Gong, and Y. Liu, Revealing the Sub-Barrier Phase Using a Spatiotemporal Interferometer with Orthogonal Two-Color 
Laser Fields of Comparable Intensity, Phys. Rev. Lett. 119, 073201 (2017).

[27] P. Eckle, A. N. Pfeiffer, C. Cirelli, A. Staudte, R. Dörner, H. G. Muller, M. Büttiker, and U. Keller, Attosecond Ionization and Tunneling Delay Time Measurements in Helium, Science 322, 1525 (2008).

[28] N. Eicke and M. Lein, Attoclock with Counter-rotating Bicircular Laser Fields, Phys. Rev. A 99, 031402(R) (2019).

[29] C. A. Mancuso, D. D. Hickstein, P. Grychtol, R. Knut, O. Kfir, X.-M. Tong, F. Dollar, D. Zusin, M. Gopalakrishnan, C. Gentry, E. Turgut, J. L. Ellis, M.-C. Chen, A. Fleischer, O. Cohen, H. C. Kapteyn, and M. M. Murnane, StrongField Ionization with Two-Color Circularly Polarized Laser Fields, Phys. Rev. A 91, 031402(R) (2015).

[30] C. A. Mancuso, D. D. Hickstein, K. M. Dorney, J. L. Ellis, E. Hasović, R. Knut, P. Grychtol, C. Gentry, M. Gopalakrishnan, D. Zusin, F. J. Dollar, X.-M. Tong, D. B. Milošević, W. Becker, H. C. Kapteyn, and M. M. Murnane, Controlling Electron-Ion Rescattering in Two-Color Circularly Polarized Femtosecond Laser Fields, Phys. Rev. A 93, 053406 (2016).

[31] A.S. Kheifets, The Attoclock and the Tunneling Time Debate, J. Phys. B 53, 072001 (2020).

[32] N. Camus, E. Yakaboylu, L. Fechner, M. Klaiber, M. Laux, Y. Mi, K. Z. Hatsagortsyan, T. Pfeifer, C. H. Keitel, and R. Moshammer, Experimental Evidence for Quantum Tunneling Time, Phys. Rev. Lett. 119, 023201 (2017).

[33] M. Klaiber, K. Z. Hatsagortsyan, and C. H. Keitel, Tunneling Dynamics in Multiphoton Ionization and Attoclock Calibration, Phys. Rev. Lett. 114, 083001 (2015).

[34] N. Fedorov et al., Aurore: A Platform for Ultrafast Sciences, Rev. Sci. Instrum. 91, 105104 (2020).

[35] M. Wollenhaupt, M. Krug, J. Köhler, T. Bayer, C. SarpeTudoran, and T. Baumert, Three-Dimensional Tomographic Reconstruction of Ultrashort Free Electron Wave Packets, Appl. Phys. B 95, 647 (2009).

[36] P. Agostini, F. Fabre, G. Mainfray, G. Petite, and N. K. Rahman, Free-Free Transitions Following Six-Photon Ionization of Xenon Atoms, Phys. Rev. Lett. 42, 1127 (1979).

[37] S. Heuser, Á. J. Galán, C. Cirelli, C. Marante, M. Sabbar, R. Boge, M. Lucchini, L. Gallmann, I. Ivanov, A. S. Kheifets, J. M. Dahlström, E. Lindroth, L. Argenti, F. Martín, and U. Keller, Angular Dependence of Photoemission Time Delay in Helium, Phys. Rev. A 94, 063409 (2016).

[38] L. Nahon, L. Nag, G. A. Garcia, I. Myrgorodska, U. Meierhenrich, S. Beaulieu, V. Wanie, V. Blanchet, R. Géneaux, and I. Powis, Determination of Accurate Electron Chiral Asymmetries in Fenchone and Camphor in the VUV Range: Sensitivity to Isomerism and Enantiomeric Purity, Phys. Chem. Chem. Phys. 18, 12696 (2016).

[39] C. Lux, M. Wollenhaupt, C. Sarpe, and T. Baumert, Photoelectron Circular Dichroism of Bicyclic Ketones from Multiphoton Ionization with Femtosecond Laser Pulses, ChemPhysChem 16, 115 (2015).

[40] C. Lux, A. Senftleben, C. Sarpe, M. Wollenhaupt, and T. Baumert, Photoelectron Circular Dichroism Observed in the Above-Threshold Ionization Signal from Chiral Molecules with Femtosecond Laser Pulses, J. Phys. B 49, 02LT01 (2016).
[41] C. F. de Morisson Faria and A. S. Maxwell, It Is All about Phases: Ultrafast Holographic Photoelectron Imaging, Rep. Prog. Phys. 83, 034401 (2020).

[42] Y. Huismans et al., Time-Resolved Holography with Photoelectrons, Science 331, 61 (2011).

[43] G. Porat, G. Alon, S. Rozen, O. Pedatzur, M. Krüger, D. Azoury, A. Natan, G. Orenstein, B. D. Bruner, M. J. J. Vrakking, and N. Dudovich, Attosecond Time-Resolved Photoelectron Holography, Nat. Commun. 9, 2805 (2018).

[44] M. Meckel, D. Comtois, D. Zeidler, A. Staudte, D. Pavičić, H. C. Bandulet, H. Pépin, J. C. Kieffer, R. Dörner, D. M. Villeneuve, and P. B. Corkum, Laser-Induced Electron Tunneling and Diffraction, Science 320, 1478 (2008).

[45] C. I. Blaga, J. Xu, A. D. DiChiara, E. Sistrunk, K. Zhang, P. Agostini, T. A. Miller, L. F. DiMauro, and C. D. Lin, Imaging Ultrafast Molecular Dynamics with Laser-Induced Electron Diffraction, Nature (London) 483, 194 (2012).

[46] K. Amini et al., Imaging the Renner-Teller Effect Using Laser-Induced Electron Diffraction, Proc. Natl. Acad. Sci. U.S.A. 116, 8173 (2019).

[47] O. Neufeld and O. Cohen, Optical Chirality in Nonlinear Optics: Application to High Harmonic Generation, Phys. Rev. Lett. 120, 133206 (2018).

[48] P. V. Demekhin, A. N. Artemyev, A. Kastner, and T. Baumert, Photoelectron Circular Dichroism with Two Overlapping Laser Pulses of Carrier Frequencies $\omega$ and $2 \omega$ Linearly Polarized in Two Mutually Orthogonal Directions, Phys. Rev. Lett. 121, 253201 (2018).

[49] A. S. Maxwell, A. Al-Jawahiry, T. Das, and C. F. d. M. Faria, Coulomb-Corrected Quantum Interference in AboveThreshold Ionization: Working towards Multitrajectory Electron Holography, Phys. Rev. A 96, 023420 (2017).

[50] T.-M. Yan and D. Bauer, Sub-Barrier Coulomb Effects on the Interference Pattern in Tunneling-Ionization Photoelectron Spectra, Phys. Rev. A 86, 053403 (2012).

[51] W. H. Press, S. A. Teukolsky, W. T. Vetterling, and B. P. Flannery, Numerical Recipes 3rd Edition: The Art of Scientific Computing, 3rd ed. (Cambridge University Press, Cambridge, England, 2007).

[52] J. Muth-Böhm, A. Becker, and F. H. M. Faisal, Suppressed Molecular Ionization for a Class of Diatomics in Intense Femtosecond Laser Fields, Phys. Rev. Lett. 85, 2280 (2000).

[53] D. Pavičić, K. F. Lee, D. M. Rayner, P. B. Corkum, and D. M. Villeneuve, Direct Measurement of the Angular Dependence of Ionization for $\mathrm{N}_{2}, \mathrm{O}_{2}$, and $\mathrm{CO}_{2}$ in Intense Laser Fields, Phys. Rev. Lett. 98, 243001 (2007).

[54] O. Smirnova, S. Patchkovskii, Y. Mairesse, N. Dudovich, and M. Y. Ivanov, Strong-Field Control and Spectroscopy of Attosecond Electron-Hole Dynamics in Molecules, Proc. Natl. Acad. Sci. U.S.A. 106, 16556 (2009).

[55] Y. Mairesse, J. Higuet, N. Dudovich, D. Shafir, B. Fabre, E. Mével, E. Constant, S. Patchkovskii, Z. Walters, M. Y. Ivanov, and O. Smirnova, High Harmonic Spectroscopy of Multichannel Dynamics in Strong-Field Ionization, Phys. Rev. Lett. 104, 213601 (2010).

[56] M. Meckel, A. Staudte, S. Patchkovskii, D. M. Villeneuve, P. B. Corkum, R. Dörner, and M. Spanner, Signatures of the Continuum Electron Phase in Molecular Strong-Field Photoelectron Holography, Nat. Phys. 10, 594 (2014). 
[57] V.S. Popov, Tunnel and Multiphoton Ionization of Atoms and Ions in a Strong Laser Field (Keldysh Theory), Phys. Usp. 47, 855 (2004).

[58] O. Smirnova and M. Ivanov, in Attosecond and XUV Physics (Wiley, New York, 2014), Chap. 7, pp. 201-256, 10.1002/9783527677689.ch7.

[59] A. Wang, Y. Zheng, C. C. Martens, and W. Ren, Quantum Tunneling Dynamics Using Entangled Trajectories: General Potentials, Phys. Chem. Chem. Phys. 11, 1588 (2009).

[60] S. Eckart, M. Kunitski, M. Richter, A. Hartung, J. Rist, F. Trinter, K. Fehre, N. Schlott, K. Henrichs, L. P. H. Schmidt, T. Jahnke, M. Schöffler, K. Liu, I. Barth, J. Kaushal, F. Morales, M. Ivanov, O. Smirnova, and R. Dörner, Ultrafast Preparation and Detection of Ring Currents in Single Atoms, Nat. Phys. 14, 701 (2018).

[61] S. Beaulieu, A. Comby, D. Descamps, B. Fabre, G. A. Garcia, R. Géneaux, A. G. Harvey, F. Légaré, Z. Mašín, L. Nahon, A. F. Ordonez, S. Petit, B. Pons, Y. Mairesse,
O. Smirnova, and V. Blanchet, Photoexcitation Circular Dichroism in Chiral Molecules, Nat. Phys. 14, 484 (2018).

[62] H. Ni, U. Saalmann, and J.-M. Rost, Tunneling Exit Characteristics from Classical Backpropagation of an Ionized Electron Wave Packet, Phys. Rev. A 97, 013426 (2018).

[63] M. E. Rose and B. T. Feld, Elementary Theory of Angular Momentum, Phys. Today 10, No. 11, 30 (1957).

[64] B. Pons, Monocentric Close-Coupling Expansion to Provide Ejected Electron Distributions for Ionization in Atomic Collisions, Phys. Rev. Lett. 84, 4569 (2000).

[65] B. Pons, Ability of Monocentric Close-Coupling Expansions to Describe Ionization in Atomic Collisions, Phys. Rev. A 63, 012704 (2000).

[66] B. Pons, Spheroidal Close-Coupling Scheme to Describe Ionization Processes in One-Electron Diatomic Systems, Phys. Rev. A 67, 040702(R) (2003). 